

\title{
Ultrahigh-Intensity Laser: Physics of the Extreme on a Tabletop
}

\author{
Gérard A. Mourou, ${ }^{1}$ Christopher P. Barty, ${ }^{2}$ Michael D. Perry ${ }^{3}$ \\ ${ }^{1}$ Center for Ultrafast Optical Science, University of Michigan, 1006 I.S.T., 2200 Bonisteel Blvd., Ann Arbor, MI \\ 48109-2099; phone: 313-763-4875; fax: 313-763-4876 \\ ${ }^{2}$ Institute for Nonlinear Science, University of California, San Diego, Urey Hall Addition 3050 G, La Jolla, CA \\ 92093-0339; phone: 619-534-2417; fax: 619-534-7654 \\ ${ }^{3}$ Lawrence Livermore National Laboratory L 439, P.O. Box 808, Livermore, CA 94550; phone: 510-423-4915; fax: \\ 510-422-5537
}

\section{Introduction}

Advance in science is often triggered by technological breakthroughs. Over the past 10 years, 1 laser intensities have increased by more than four orders of magnitude to reach enormous intensities of $10^{20} \mathrm{~W} / \mathrm{cm}^{2}$ (Figure 1). The field strength at these intensities is of the order of a teravolt $/ \mathrm{cm}$, or 100 times the Coulombic field that binds the electrons to the nucleus. The electrons driven by such a field are relativistic, with an oscillatory energy of $10 \mathrm{MeV}$. At these intensities, the light pressure, $P=2 I / c$, is extreme, of the order of giga- to terabars. The laser interacting with matter-gas, solid, plasma-will generate high-order harmonics, energetic ions or electrons, gigagauss magnetic fields, and violent accelerations of $10^{20} g$, where $g$ is earth gravity. Finally, the interaction of an ultraintense beam with superrelativistic particles can produce fields greater than critical and nonlinear quantum electrodynamical effects can be observed. In many ways, this physical environment of extreme fields, pressure, magnetic field, temperature, and acceleration can only be found in stellar interiors or close to the horizon of a black hole and it is fascinating to think that an astrophysical environment governed by hydrodynamics, radiation transport, and gravitational interaction could be re-created in university laboratories for an extremely short time, switching the role of scientists from voyeurs to actors.

Also, ultrahigh-intensity lasers will play an important role in the development of new compact, tabletop-size, ultrashort sources of high-energy photons ( $x$ ray) and electrons (giga-electronvolt) with attosecond duration. The period of light being $3 \mathrm{fs}$ at $1 \mu \mathrm{m}$, getting into the attosecond regime will require shorter wavelengths, which naturally involves high intensities. For instance, short wavelengths can be produced by nonlinear effects like harmonic generation and produce subfemtosecond pulses. Similarly, the enormous field gradients that can be produced in laser plasma interaction can accelerate electrons over a distance $10^{4}$ times shorter than what was previously possible and the resulting electron pulses are expected to be well in the subfemtosecond range. These ultrafast sources will have important applications for time resolving the structure of matter at the atomic or molecular level. Short pulses are necessary, 
considering that the atomic motion is in the 100-fs range. Ultrahigh intensity led to a dramatic extension to the giga-electron-volt range of laser applications, until then confined to the $1-10-\mathrm{keV}$ regime.

What is spectacular is that the size of the laser involved is extremely compact (tabletop) and relatively inexpensive. It can deliver high average power and be built and operated by students.

Some of these tabletop-laser principles can be easily implemented at a minimum cost on existing large laser systems already built for laser fusion. Lawrence Livermore Laboratory, ${ }^{1} \mathrm{CEA}-$ Limeil, ${ }^{1}$ and Osaka ${ }^{1}$ have converted their nanosecond lasers to subpicosecond pulse duration, pushing their peak power by 3 orders of magnitude from the terawatt $\left(10^{12}\right)$ to $100-1000 \mathrm{TW} .1$ They also can be moved to very large structures like synchrotrons (ALS, ESRF) to produce ultrashort, high-energy $x$ rays by Compton scattering, or to accelerator facilities like SLAC to test nonlinear quantum electrodynamics by the interaction of the high-intensity pulses with superrelativistic electrons.

Figure 1 shows laser intensity as a function of years. We can see a rapid increase in intensity in the early $1960 \mathrm{~s}$, followed by a long plateau at $10^{15} \mathrm{~W} / \mathrm{cm}^{2}$. It took about 20 years, until 1987, for laser power to increase again. It is important to stress the similarity in slopes between the early 1960s and the past ten years, and to remember that it is during this period of very rapid increase in intensities in the 1960s that most of the nonlinear optics phenomena were discovered. In a similar way, the spectacular increase in intensity (four to five orders of magnitude) that we are experiencing today should lead tó exceptional scientific discoveries. Finally, from an educational point of view, this compact laser offers the advantage of bringing some of the research traditionally done on large instruments to human-size setups in university laboratories. Their small scale and large numbers should greatly foster diversification and multidisciplinarity and should attract students to scientific disciplines.

\section{The Evolution of Laser Peak Power}

Although strong-field physics with conventional lasers had already started in the $1960 \mathrm{~s}^{2}$ the scientific revolution started about 10 years ago. At that time, we demonstrated that ultrashort pulses at the 100-fs level could be amplified, without damaging the amplifying media, to the terawatt level, using a technique that we called Chirped Pulse Amplification (CPA). ${ }^{3}$

Since their inception in 1960, the peak power of lasers has evolved by a succession of leaps of three orders of magnitude. They were produced each time by decreasing the pulse duration accordingly. First the lasers were free running, with a duration in the $10-\mu$ s and peak power in the kilowatt range. In 1962, modulation of the laser-cavity quality factor allowed the same 
energy to be released in a nanosecond time scale, a thousand times shorter, to produce pulses in the megawatt range. In 1964, locking the longitudinal modes of the laser (mode locking) allowed the laser pulse duration to be reduced by another factor of a thousand, down to the picosecond level, pushing the peak power a thousand times higher, to the gigawatt level. At this point, the intensities associated with the ultrashort pulses were becoming prohibitively high-i.e., in the $\mathrm{GW} / \mathrm{cm}^{2}$ range. At these intensities, the index of refraction becomes linearly dependent on the intensity to vary like $n=n_{0}+n_{2} I$, where $n$ is the index of refraction, $n_{0}$ the index of refraction at low intensity, $n_{2}$ the nonlinear index of refraction, and $I$ the intensity. The result is that, for a beam with a Gaussian radial intensity distribution, the center of the beam sees a larger index of refraction than its sides. The material becomes a positive lens and alters the beam wave-front quality to an unacceptable level, to the point where it can create filaments and irreversible damage to the laser system. The only way to increase the peak power was to increase the beam diameter at the expense of size, low repetition rate, and cost.

Although the pulse duration kept decreasing, for about 20 years the intensity limitation in laser systems kept the peak power about constant at the gigawatt level for a $\mathrm{cm}^{2}$ beam, until 198587, when the technique of CPA was demonstrated. The CPA concept is illustrated in Fig. 2 . The short pulse is first produced by an oscillator. After generation it is not amplified directly, but stretched by a large amount, $10^{3}$ to $10^{5}$, from femtosecond to nanosecond, reducing the intensity accordingly. The pulse intensity is now low enough that the stored energy can be safely extracted out of the amplifier, without fear of beam distortions and damage. Once the stored energy is fully extracted, the pulse is recompressed, ideally to its initial value. The key point of the CPA technique is that it decouples pulse fluence (energy $/ \mathrm{cm}^{2}$ ) and pulse intensity (power $/ \mathrm{cm}^{2}$ ). So it reconciles two apparently conflicting needs: to have (a) the highest fluence for efficient energy extraction and (b) minimum intensity to avoid the undesired nonlinear effects.

CPA had a dramatic impact. First, we could for the first time use extremely good energy storage media like Nd:glass, ${ }^{3}$ alexandrite, ${ }^{1}$ Ti:sapphire, ${ }^{1}$ and $\mathrm{Cr}: \mathrm{LiSAF}^{1}{ }^{1}$ and extract their energy fully. Before CPA, dyes and excimers, which are a thousand times inferior in energy storage, were the only choice for ultrashort pulse generation. So at equal size, a CPA laser system, using the superior energy storage media, could produce $10^{3}$-to- $10^{4}$ times higher peak power than conventional dye or excimer systems. Second, CPA could be easily adapted on very large-scale, expensive lasers already built for laser fusion. By simple beam manipulations, a stretching at the beginning and a compression at the end of the amplifying chain, these expensive systems built to amplify nanosecond pulses to the terawatt level could be converted to amplify femtosecond pulses, as well, and to produce petawatt pulses. 
It is interesting to note that this sudden improvement by three-to-four orders of magnitude is similar to the one in electronics in 1960-when they went from discrete $(\mathrm{mm})$ to integrated $(\mu \mathrm{m})$ circuits; or to the advent of laser diodes-where meter-size lasers could be miniaturized to millimeters. As in electronics, this enormous reduction in size led to new advantages: speed, efficiency, reliability, and cost.

\section{Unleashing Laser Power: Chirped Pulse Amplification (CPA)}

CPA (Fig. 2) involves impressive manipulations. Stretching by $10^{3}-10^{5}$, amplification by $10^{11}$ (nanojoules to tens of joules), and recompression by $10^{3}$ to $10^{5}$. Because we want the highest intensities on target, it is important that these manipulations be performed with the highest degree of fidelity, so that the pulse in the time and spatial domains possesses the highest temporal contrast and most of the energy will be in the diffraction-limited spot. Two main hurdles had to be overcome for the generation of ultrahigh peak power: (a) the accommodation of the large stretching-compression ratio and (b) the amplification of large pulse spectra.

\section{a. Clearing the First Hurdle: Discovery of the Matched Stretcher}

We may wonder how we can stretch and compress a short pulse over so many orders of magnitude with impunity and fidelity. This came with the discovery of the matched stretchercompressor. The first CPA system ${ }^{3}$ was using the positive group velocity dispersion of a singlemode fiber. At that time, the only practical way we knew, to stretch a pulse was with positive group velocity dispersion (where the red wavelength goes faster than the blue). Once it had been amplified, the only way we knew to compress the pulse was to use a pair of parallel gratings. Such a pair exhibits a negative group velocity dispersion, as demonstrated by Treacy in $1969^{1}$ (blue goes faster than the red, Fig. 2a), so the pulse could be put back together. However, the stretcher and compressor did not have their dispersive characteristics perfectly matched, so the recompression was not perfect, leading to temporal wings in the pulse, hence limiting the stretching-compression ratio to about 100 .

We became aware of a "compressor" designed by O. Martinez, ${ }^{4}$ built for optical communication to compress pulses at $1500 \mathrm{~nm}$. We recall that in this spectral range, light experiences a negative group velocity dispersion. This new dispersive system was composed of a telescope of magnification of one between two antiparallel gratings (Fig. 2b). Studying this system, it became clear to us that it had the exact same dispersive function as the Treacy compressor, but with the opposite sign. In other words, it was the perfect conjugate of the Treacy compressor-with the important consequence that any arbitrary short pulse could in theory be stretched to any pulse duration and put back to its original shape. A stretching factor of $10^{3}-10^{4}$ was immediately demonstrated with a Nd:glass system, to produce the first tabletop terawatt laser system. ${ }^{3}$ 
With the discovery of the matched stretcher-compressor, a very high hurdle was cleared. For the amplification of pulses at the 10-fs level, stretching up to $10^{5}$ can be required ${ }^{5}$ In this case, it is necessary to design the expander not to match the compressor alone but instead to match the sum of the phase function of the gain medium, mirrors, and other optical elements in the amplification chain. The other alternative, used by Kapteyn et al., 5 is to use very short-length amplifying media and to minimize the stretching ratio. Due to the very high peak power, the pulse after compression must be in vacuum to avoid nonlinear effects in air ${ }^{14}$ that will produce wave-front distortion and filamentation.

\section{b. Clearing the Second Hurdle: The Discovery of Ultrabroadband Gain Media}

Ultrashort pulses have enormous spectra. From the uncertainty principle $\Delta v \cdot \tau=0.35$, where $\Delta v$ is the pulse spectrum and $\tau$ is the pulse duration, a 10 -fs pulse will have a bandwidth of $70 \mathrm{~nm}$. All the spectral components will have to be equally amplified over many orders of magnitude at the risk of narrowing its bandwidth and lengthening its duration. For ultrashort pulses, this limitation was removed with the invention of ultrabroadband amplification media, such as Ti:sapphire ${ }^{6}$ and Cr:LiSAF, 7 and with the development of techniques such as the regenerative pulse shaping introduced by Barty et al..$^{5}$ to eliminate gain narrowing during the amplification process. In particular, Ti:sapphire, whose gain bandwidth can theoretically support the amplification of pulses of less than $5 \mathrm{fs}$ in duration, is ideally suited for CPA because of its excellent thermal and optical properties and moderate saturation fluence. Because of the excellent thermal properties, ultrashort pulses could be amplified at a much larger repetition rate, $10-10^{3} \mathrm{~Hz}$, bringing the average power of Ti:sapphire-based CPA to the watt level. 8,5 This represents an improvement of 2-3 orders of magnitude over conventional amplification systems based on dyes or excimers. Increased repetition rate at constant peak power leads directly to higher experimental utility. With ultrafast CPA systems, for instance, it is possible to apply signal-averaging techniques to high-field laser/matter investigations.

At the University of California, San Diego, 4-TW, sub-20-fs FWHM pulses ${ }^{5}$ have been demonstrated from a Ti:sapphire amplification chain operating at a $10-\mathrm{Hz}$ repetition rate. This laser is shown in Fig. 4. At ENSTA, France, the University of Tokyo, and the Japanese Atomic Energy Research Institute, ${ }^{5}$ 30-TW, 20-30-fs pulses ${ }^{5}$ have been obtained. Extension of these concepts to higher peak power and higher repetition rate are straightforward and are currently under development. In Japan, the Kansai research establishment of the Japanese Atomic Energy Research Institute is presently designing and testing components for a petawatt (20-J, 20-fs), 1-Hz laser which is scheduled for construction in 1999. In Bordeaux, France, and at the NSF Center for Ultrafast Optical Science (CUOS) at the University of Michigan, funds have been 
allocated and designs completed for the construction a 1-TW (20-mJ, 20-fs), 1-kHz laser facilities.

\section{The Generation of Ultrashort Pulses and Their Characterization}

Ultrashort pulses as short as a few optical cycles have been with us for 10 years. But it is the outstanding discovery by W. Sibbett in 1991 of Kerr Lens Mode Locking (KLM) ${ }^{9}$ with its successive refinements ${ }^{11}$ that made the generation of pulses shorter than 10 fs routine. These systems will provide a robust source of short seed pulses at the nanojoule level for CPA systems.

The concept of the generation of ultrashort pulses by KLM is simple and uses simultaneously the very large bandwidth of Ti:sapphire and its optical Kerr effect, or the intensity-dependent index of refraction, described above. A simplified diagram of a laser cavity is shown in Fig. $3 a$. The cavity is composed of curved mirrors and the amplifying medium (Ti:sapphire). The amplifier is optically pumped, usually with a continuous-wave argon laser. The Ti:sapphire has a double role. The first is to amplify the pulse, to compensate the various losses in the cavity. The second is to be a lens with an intensity-dependent focal length. We have seen previously that the Gaussian beam profile will produce a positive lens due to the intensity-dependent index of refraction. So the experimentalist adjusts the wave-front radius of curvature to be equal to one of the mirrors for the highest intensity or the shortest pulse duration. In other words, it is where the laser experiences the minimum amount of losses that the laser will run. To get to the ultimate pulse duration, the cavity mirrors (no lenses to avoid dispersive effects) must be broadband enough and the dispersion of the pulse in crystal must be compensated 10 by frequency-chirped mirrors (Krausz and coworkers) or by a sequence of prisms (Murnane and Kapteyn group). Pulses down to $6.5 \mathrm{fs}$ have been produced directly out of the oscillator by U. Keller et al. 10

\section{The Petawatt: The Heavyweight CPA}

The Petawatt Project, to explore the feasibility of the fast ignition concept, was realized at Livermore by one of the authors. It illustrates beautifully the adaptability of CPA. They use a beam of the large NOVA, a Nd:glass laser chain built for nanosecond pulse amplification delivering kilojoules in a few nanoseconds. Although conceptually simple, the adaptation of CPA on this laser chain required the development of sophisticated new technology: in particular, the design of very large diffraction gratings with $75-\mathrm{cm}$ diameter, efficiency greater than $90 \%$, with flatness-better than $\lambda / 10$, and a good damage threshold. Thus, $1.3 \mathrm{~kJ}$ could be extracted in $800 \mathrm{ps}$ and compressed in $430 \mathrm{fs}$, producing $1.3 \mathrm{PW}$ with $>10^{21} \mathrm{~W} / \mathrm{cm}^{2}$ 
irradiance 11 - the highest peak power and irradiance ever produced. Currently, the petawatt operates with a $46-\mathrm{cm}$ beam and $75-\mathrm{cm}$ grating. These large gratings forming the compressor were mounted in a vacuum chamber to avoid self-focusing in air. ${ }^{14}$ These gratings were increased to the full $94-\mathrm{cm}$ size in July 1997, thereby allowing operation of the petawatt at $1 \mathrm{~kJ}$ with a $58-\mathrm{cm}$ diameter beam.

\section{How High Can the Peak Power Go?}

Is it possible to predict the ultimate peak power that we will able to achieve for a given-size system? A good estimate can be obtained using the following simple argument. The maximum energy that can be extracted from an amplifier is of the order of the saturation fluence, $F_{s}=\frac{h v}{\sigma}$, where $\sigma$ is the transition cross section, while the shortest pulse duration $\tau$ is limited by the gain bandwidth of the amplifying medium, $\Delta v$. The maximum theoretical peak power per $\mathrm{cm}^{2}$ of beam is therefore given simply by $P_{t h}=\frac{h v \Delta v}{\sigma}$. It is, in fact, the intensity necessary to flip the population in a time corresponding to the dephasing time of the transition. This quantity is known in quantum electronics as the Rabi intensity. The maximum focusable intensity will then be equal to this power over a spot size corresponding to $\lambda^{2}$. The highest intensity will therefore be $I_{t h}=\frac{h v^{3} \Delta v}{c^{2} \sigma}$. The highest peak power will be produced by amplifying media with the smallest transition cross section and the largest bandwidth.

Here are some examples. $P_{\text {th }}$ varies from 200 TW for Ti:sapphire to 3000 TW for $\mathrm{Yb}$ :glass. Using Yb:glass, a material that can be obtained in large dimensions, a system with a beam size of $10 \mathrm{~cm}$ by $10 \mathrm{~cm}$ could produce peak power of $0.3 \mathrm{EW}$ (an exawatt is $10^{18} \mathrm{~W}$ ). This power, focused over a diffraction-limited spot size of a few $\mu \mathrm{m}^{2}$, could produce on-target intensities in the $10^{24} / \mathrm{cm}^{2}$ range!

\section{Ultrahigh-Intensity Applications}

Figure 1 shows the laser intensity and the different physical regimes that are becoming accessible. Because of the intense activities that this field experiences, it is impossible in an exhaustive manner to do justice to all contributors. We will stress in the following only some of the most recent works and directions that occurred over the past few years.

\section{At $10^{14} \mathrm{~W} / \mathrm{cm}^{2}$}

High harmonic generation from gases ${ }^{2}$ is still a fascinating and active topic. The bound electrons are driven by the high field. Their response is highly nonlinear and harmonics of the laser to very large orders are emitted. These harmonics can be generated up to a cutoff frequency ${ }^{2}$ given by 
$h v_{n}=E_{i}+3.2 U_{p}$, where $h$ is the Planck constant, $v_{n}$ the harmonic frequency, $E_{i}$ the ionization energy, and $U_{p}$ the quiver energy. It was recently found that by shortening the pulse to a few optical periods, $10-20 \mathrm{fs}$, higher ionization energy could be obtained, ${ }^{12}$ pushing the harmonic number over 300 -that is, well within the water window. ${ }^{13}$ An interesting aspect of high harmonic generation is that it may provide a way to generate pulses of attosecond duration. At these intensities, when the main pulse freely propagates, due to the combination of diffraction, intensity-dependent index of refraction $\left(n_{2}\right)$, and field ionization, it can condense into "light bullets $^{\prime \prime 14}$ of $80-\mu \mathrm{m}$ diameter that can propagate over distances greater than $100 \mathrm{~m}$. Important applications of this channeling are currently being investigated: guiding for efficient harmonic generation in gases and electron acceleration, and control of lightning for airport and nuclear plant protection as proposed by J. C. Diels. ${ }^{14}$ In solids at $10^{15} \mathrm{~W} / \mathrm{cm}^{2}$, a plasma forms, light is absorbed, and material damaged. First, the free electrons are generated by single- or multiphoton absorption. They are then multiplied to reach the critical density where the plasma can absorb the laser energy, due to the great number of collisions $\left(10^{15} \mathrm{~Hz}\right)$, to finally damage the material. Because the single- or multiphoton absorption is a deterministic ${ }^{15,16}$ process and the impact ionization as a function of the field saturates, ablation with ultrashort pulses becomes very deterministic (accurate threshold)-as opposed to stochastic for long pulses. This deterministic character may lead to some of the most important practical applications of ultrashort pulses: eye surgery, micromachining, and data storage. 16

Intensities $10^{16} \mathrm{~W} / \mathrm{cm}^{2}$

Laser interaction with clusters is becoming very popular and shows enhanced harmonic generation and $x$-ray production, as well as a copious amount of high-energy electrons up to $3 \mathrm{keV}$ and ions up to $1 \mathrm{MeV} .{ }^{17}$ In solids, 18 the combination of high electron temperature and large ponderomotive pressure, which at these intensities become comparable to the thermal pressure (see the work of Umstadter et al. ${ }^{2}$ ), contribute to increase the mean free path. As a result, collisional absorption is suppressed to give rise to collisionless absorption like resonance absorption or by anomalous skin effect, when the collision mean free path becomes larger than the skin depth.

The electrons driven through steep gradients can radiate very high harmonics. Up to the 70 th harmonic was produced with a 2.5-ps CPA laser. ${ }^{18}$ Harmonic generation in solids is very sensitive to prepulse, which affects the gradient steepness. Very high harmonics, order $n>100$, should be produced with good efficiency $\left(>10^{-5}\right)$ with shorter $(100-\mathrm{fs})$ and cleaner pulses (see Von der Linde ${ }^{19}$ ). 


\section{Intensities $>10^{18} \mathrm{~W} / \mathrm{cm}^{2}$}

At these intensities, the electrons in the field of the laser oscillate with a quiver velocity near the speed of light (quiver energy becomes equal to the rest mass, i.e., $0.5 \mathrm{MeV}$ ), and mass increases by the relativistic factor $\gamma$. For light at $1 \mu \mathrm{m}, \gamma$ equals $\sqrt{2}$. The force applied to the electron is expressed by the Lorentz equation $F=q \cdot\left(E+\frac{v}{c} \wedge B\right)$, where $q$ is the charge of the electron, $E$ and $B$ the electric and magnetic fields) of the laser, $v$ the quiver velocity, and $c$ the speed of light. In conventional linear or nonlinear optics, the force due to the magnetic field is always neglected due to the small quiver velocity of the electron compared to $c$. However, at $10^{18} \mathrm{~W} / \mathrm{cm}^{2}, v$ is close to $c$, and the magnetic and electric force become equal, opening up the new field of relativistic nonlinear optics. This regime is characterized by extremely large-greater than gigabar-light pressure, much larger than the thermal pressure. Because the laser is focused on a very small spot size of $10 \mu \mathrm{m}$ (several wavelengths), a large lateral gradient will be produced that will have the tendency to push the electrons from the center of the focal spot, dragging the more massive ions with them. 20 The beam center will be evacuated, cavitation 20 decreasing both ion and electron densities. The simultaneous decrease of the electron density and plasma frequency $\omega_{p}=\frac{\omega_{p o}}{\gamma}$, where $\omega_{p o}$ is the plasma frequency without mass correction, will lead to induced transparency. That may have been observed recently by Giuletti et al. ${ }^{19}$

In subcritical gases, this enormous ponderomotive pressure produced by a focused pulse can displace the electrons in its way. The electrons will be repelled by the ions once the pulse goes by, 21 producing a large plasma wave with electrostatic fields greater than $200 \mathrm{GeV} / \mathrm{m}$ or $10^{4}$ larger than what can be produced with conventional technologies. This concept proposed by Tajima and Dawson is known as laser wakefield acceleration. ${ }^{21}$ Collimated electron beams of a few nanocoulombs with mega-electron-volt energy have been produced by this simple technique, ${ }^{22}$ where the laser pulse is focused onto a gas jet $1 \mathrm{~mm}$ thick (Fig. 5). A scheme to inject electrons in the narrow acceleration bucket of about 50 fs has been proposed by Umstadter. ${ }^{22}$ Because the electrons become immediately relativistic, the space charge broadening will be minimum. According to simulations, subfemtosecond pulses of electrons could be produced. At these intensities, the plasma frequency, which is intensity-dependent due to the mass change, will vary across the laser spot size, altering the index of refraction accordingly, causing the laser to self-focus to a $\mu \mathrm{m}$-size filament. ${ }^{21}$ This is known as relativistic self-focusing, predicted by Litvack and Max ${ }^{21}$ in the 1970s. A large current density, of the order of $10^{12} \mathrm{~A} / \mathrm{cm}^{2}$, will circulate in the filament to produce an extremely large magnetic field of gigagauss strength. 20 
In wakefield acceleration, electrons are accelerated from a cold start to $c$ in a fraction of a picosecond corresponding to $10^{21} \mathrm{~g}$ that could lead to detectable Unruh radiation. ${ }^{23} \mathrm{~A}$ recent experiment on solid targets ${ }^{19}$ by Saubrey et al. has already confirmed plasma acceleration of $10^{17} \mathrm{~g}$ with a modest intensity by today's standard of $10^{18} \mathrm{~W} / \mathrm{cm}^{2}$. This kind of acceleration is the one found near the horizon of a black hole. This result illustrates the exciting possibility for ultrahigh intensity to test general relativity in a laboratory setting.

In solids, one of the most impressive applications is the so-called Fast Ignitor. ${ }^{1}$ The essential idea, as illustrated in Fig. 6, is to pre-implode a deuterium-tritium capsule to an isochoric (uniform-density) condition (see J. C. Kieffer et al. ${ }^{1}$ ). At the point of maximum compression, irradiate the side of the imploded core with a laser pulse much shorter than the hydrodynamic disassembly time of the irradiated spot $\left(\tau \approx \frac{R_{\text {spot }}}{v_{s}} \leq 10 \mathrm{ps}\right)$. Hot electrons $(200 \mathrm{keV}<E<1$ $\mathrm{MeV})$ generated by the interaction of the intense $\left(10^{19}-10^{21} \mathrm{~W} / \mathrm{cm}^{2}\right)$ light with plasma rapidly equilibrate in the dense fuel. The energy equilibration of the electrons raises the overall ion temperature to $5-20 \mathrm{keV}$, initiating fusion burn. High laser pulse energy is required in order to produce enough hot electrons to heat a sufficient number of ions-to initiate fusion burn. In addition, nearly perfect beam quality is required in the laser in order to achieve a small spark region. The Fast Ignitor concept offers the possibility of high target gain at reduced total drive energy, compared with conventional ICF.

It has been suggested by Tajima, Takabe, Ichimaru, and Remington ${ }^{19}$ that astrophysical conditions such as opacity, densities, and temperatures could be reproduced and tested at intensities of $10^{20} \mathrm{~W} / \mathrm{cm}^{2}$, corresponding to gigabar pressures. One proposal is to look at the rates of nuclear fusion, such as thermonuclear and pycnonuclear, that are greatly modified by the state of dense matter. Ultrahigh-pressure metal physics with ultrahigh-intensity lasers could be applied to understand the phase transformation, metallization, and crystallization of hydrogen in stellar interiors and help the investigation of the physical mechanisms governing supernovae, stars, and nebulae.

\section{Nonlinear quantum electrodynamics}

The possibility of creating electron-positron pairs from the laser field was proposed in the late 1960s. As seen in Fig. 1, pair creation directly from the laser will require intensity of the order of $10^{30} \mathrm{~W} / \mathrm{cm}^{2}$. With $E$ as the laser field, it is the energy required over the Compton wavelength, $\lambda_{c}=\frac{h}{m_{0} c}$, to create an electron-positron pair. The field is therefore equal to $E=\frac{2 m_{0} c^{2}}{e \lambda_{c}}$ and corresponds to $10^{16} \mathrm{~V} / \mathrm{cm}$, which is about four-to-five orders of magnitude above the laser field of today's laser. This enormous gap was bridged 24 by using the electric field enhancement 
produced in the frame of superrelativistic electrons. Using the $50-\mathrm{GeV}$ electron beam at the Stanford Linear Accelerator, corresponding to a $\gamma$ of $10^{5}$, with a currently available high-power laser, the field is enhanced to $E \sim 10^{16} \mathrm{~V} / \mathrm{cm}$, exceeding the critical field. The multiphoton pair production, $w_{\gamma}+n w_{0} \rightarrow e^{+}+e^{-}$, has been observed, where $w_{\gamma}$ is the upconverted frequency from Compton Scattering and $w_{0}$ is the laser frequency. The same group is studying nonlinear Compton scattering, $e+n w_{0} \rightarrow e^{\prime}+w_{\gamma}$. In this case, the high-energy $\gamma$ ray produced in the laser focus by an incident electron interacts before leaving the focal region. Up to the $n=4$ process has been observed.

Work cited above was supported by the National Science Foundation through the Center for Ultrafast Optical Science under STC PHY 8920108; by the U.S. Department of Energy through University of California Lawrence Livermore National Laboratory DOE contract W-7405-ENG-48; and by the University of California Los Alamos National Laboratory GB/020165/AL/03. 


\section{Figure Captions}

1. Laser intensity vs. years showing the different physical thresholds.

2 (a) The CPA concept, whereby an ultra short pulse is stretched by a large factor, $10^{3}-10^{5}$, amplified by $10^{11}$, and recompressed by $10^{3}-10^{5}$.

(b) The Matched Stretcher-Compressor. The stretcher is composed of a pair of telescopes of magnification of one between two anti-parallel gratings. Note that in this configuration the red wavelengths have a shorter path than the blue ones. Conversely the compressor is composed of a pair of parallel gratings where the optical length for the blue is shorter than for the red.

3 (a) A schematic of an ultrashort pulse cavity. The use of chirped mirrors makes the cavity dispersion free, in order to accommodate the very large Fourier spectrum of a sub-10femtosecond pulse.

(b) The autocorrelation trace of the output pulse produced by mixing the signal with itself in a second harmonic crystal.

4. A schematic of the 50-TW and 5-TW Ti:sapphire CPA systems (Barty et al.) ${ }^{5}$

5. The impact of a high-energy, collimated beam of electrons (up to $50 \mathrm{MeV}$ ) on a phosphor screen produced by the interaction of the ultraintense pulse with a 1-mm gas jet of He. The laser is blocked by an Al foil.

6. Diagram of fast ignition concept in inertial confinement fusion. 


\section{References}

1. M. D. Perry and G. Mourou, Science 264, 917 (1994).

2. C. Joshi and P. B. Corkum, Physics Today 48, 36-43 (1995).

3. D. Strickland and G. Mourou, Opt. Commun. 56, 219 (1985); P. Maine, D. Strickland, P. Bado, M. Pessot, and G. Mourou, IEEE J. Quantum Electron. 24, 398 (1988).

4. O. E. Martinez, ibid. 23, 1385 (1987).

5. B. E. Lemoff and C. P. Barty, Opt. Lett. 18, 1651 (1993); J. Zhou, G. Taft, C. P. Huang, M. M. Murnane, H. C. Kapteyn, I. Christov, ibid. 19, 1149 (1994); C. Barty, T. Guo, C. LeBlanc, F. Raski, C. Rose-Petruck, J. Squier, K. Wilson, V. Yakolev, and K. Yamakawa, ibid. 20, 668 (1996); J. P. Chambaret, C. LeBlanc, G. Cheriaux, P. Curley, G. Darpentigny, and P. Rousseau, ibid. 21, 1921 (1996); Japan Atomic Energy Research Institute, private communication, Watanabe, University of Tokyo; K. Yamakawa, M. Aoyama, S. Matsuoka, Y. Akahane, T. Takuma, C. P. Barty, and D. Fittinghoff, "International Conference on Superstrong Fields in Plasmas," Varenna, Italy (1997); J. Itatani, Y. Nabekawa, K. Kondo, and S. Watanabe, Opt. Commun. 134, 134 (1995).

6. P. F. Moulton, J. Opt. Soc. Am. B 3, 125 (1986).

7. S. A. Payne, L.L.Chase, L. K. Smith, W. L. Kway, and H. W. Newkirk, J. Appl. Phys. 66, 1051 (1989).

8. F. Salin, J. Squier, G. Mourou, G. Vaillancourt, Opt. Lett. 16, 1964 (1991); T. B. Norris, ibid. 17, 1009 (1992); S. Backus, C. Durfee, G. Mourou, H. C. Kapteyn, M. Murnane, Opt. Lett. 22, 1256 (1997).

9. C. V. Shank, Ultrashort Laser Pulses and Applications, ed. by W. Kaiser (Springer-Verlag, Berlin, 1988), Vol. 60, pp. 5-34; D. E. Spence, P. N. Kean, W. Sibbett, ibid. 16, 42 (1991).

10. J. Zhou, G. Taft, C. P. Huang, M. M. Murnane, H. C. Kapteyn, I. Christov, ibid. 19, 1149 (1994); Ch. Spielmann, P. F. Curley, T. Brabec, F. Krausz, IEEE J. Quantum Electron. 30, 1100 (1994); A. Stingl, Ch. Spielmann, F. Krausz, R. Szipöcs, Opt. Lett. 19, 204 (1994); I. D. Jung, F. X. Kärtner, N. Matuschek, D. H. Sutter, F. Morier-Genoud, G. Zhang, U. Keller, V. Scheuer, M. Tilsch, and T. Tschudi, ibid. 22, 1009 (1997).

11. M. D. Perry, B. C. Stuart, G. Tietbohl, J. Miller, J. A. Britten, R. Boyd, M. Everett, S. Herman, H. Nguyen, H. T. Powell, and B. W. Shore, CLEO'96 Technical Digest Series (Opt. Soc. America, Washington, DC, 1996), Vol. 9, pp. 307-308.

12. J. Zhou, J. Peatross, M. Murnane, H. Kapteyn, I. Christov, Phys. Rev. Lett. 76, 752 (1996). 
13. Krauss, CLEO 97; Z. Chang, A. Rundquist, H. Wang, H. Kapteyn, M. Murnane, to be published in Physical Review Letters (1997).

14. A. Braun, G. Korn, X. Liu, D. Du, J. Squier, and G. Mourou, Ultrafast Phenomena IX (Springer, Berlin, 1994), Vol. 60, p. 248.

15. D. Du, X. Liu, G. Korn, J. Squier, and G. Mourou, Appl. Phys. Lett. 64, 3071 (1994).

16. B. C. Stuart, M. D. Feit, A. M. Rubenchik, B. W. Shore, and M. D. Perry, Phys. Rev. Lett. 74, 2248 (1995); E. N. Glezer, M. Milosavljevic, L. Huang, R. J. Finlay, T.-H. Her, T. P. Callan, and E. Mazur, Opt. Lett. 21, 2023 (1996).

17. T. Ditmire, T. Donnelly, R. W. Falcone, and M. D. Perry, Phys. Rev. Lett. 75, 3122 (1995); T. Ditmire, J. W. G. Tisch, E. Springate, M. B. Mason, N. Hay, R. A. Smith, J. P. Marangos, and M. H. R. Hutchinson, Applications of High Field and Short Wavelength Sources VII (Opt. Soc. America, Washington, DC, 1997).

18. See, for instance, P. Gibbon and E. Förster, Plasma Phys. Control Fusion 38, 769 (1996).

19. D. von der Linde, "Generation of Ultrashort XUV Pulses by High-Order Harmonic Generation from Plasma-Vacuum Bound," "International Conference on Superstrong Fields in Plasmas," Varenna, Italy (1997); R. Sauerbrey, "Femtosecond Laser Plasmas, FROGS, and Black Holes," "International Conference on Superstrong Fields in Plasmas," Varenna, Italy (1997); A. Giulietti, "High-Intensity 30-fs Laser Pulse Interaction with Thin Foils," "International Conference on Superstrong Fields in Plasmas," Varenna, Italy (1997); H. Takabe, "Laboratory Astrophysics Using Intense and Ultraintense Lasers," "International Conference on Superstrong Fields in Plasmas," Varenna, Italy (1997); B. Remington, "Bringing the Stars Down to Earth with Lasers," "International Conference on Superstrong Fields in Plasmas," Varenna, Italy (1997); S. Ichimaru, "Ultradense Hydrogen: Frontiers in Astrophysics, Ultrahigh-Pressure Metal Physics, and Fusion Studies," "International Conference on Superstrong Fields in Plasmas," Varenna, Italy (1997).

20. A. Pukhov and J. Meyer-ter-Vehn, Phys. Rev. Lett. 7 6, 3975 (1996).

21. E. Esarey, P. Sprangle, J. Krall, and A. Ting, IEEE Trans. Plasma Sci. 24, 252 (1996).

22. A. Modena, Z. Najmudin, A. E. Dangor, C. E. Clayton, K. A. Marsh, C. Joshi, V. Malka, C. B. Darrow, C. Danson, D. Neely, and F. N. Walsh, Nature 377, 606 (1995); K. Nakajima, D. Fisher, T. Kawakubo, H. Nakanishi, A. Ogata, Y. Kato, Y. Kitagawa, R. Kodama, K. Mima, H. Shiraga, K. Suzuki, K. Yamakawa, T. Zhang, Y. Sakawa, T. Shoji, Y. Nishida, N. Yugami, M. Downer, and T. Tajima, Phys. Rev. Lett. 74, 4428 (1995); D. 
Umstadter, S.-Y. Chen, A. Maksimchuk, G. Mourou, and R. Wagner, Science 273, 472

(1996); S. Le Blanc, M. Downer, R. Wagner, S.-Y. Chen, A. Maksimchuk, G. Mourou, and D. Umstadter, Phys. Rev. Lett. 77, 5381 (1996).

23. P. Chin, and T. Tajima, to be published, Physical Review Letters.

24. C. Bula, K. T. McDonald, E. J. Prebys, C. Bamber, S. Boege, T. Kotseroglou, A. C. Melissinos, D. D. Meyerhofer, W. Ragg, D. L. Burke, R. C. Field, G. Horton-Smith, A. C. Odian, J. E. Spencer, D. Waltz, S. C. Berridge, W. M. Bugg, K. Shmakov, and W. Weidemann, Phys. Rev. Lett. 76, 3116 (1996). 


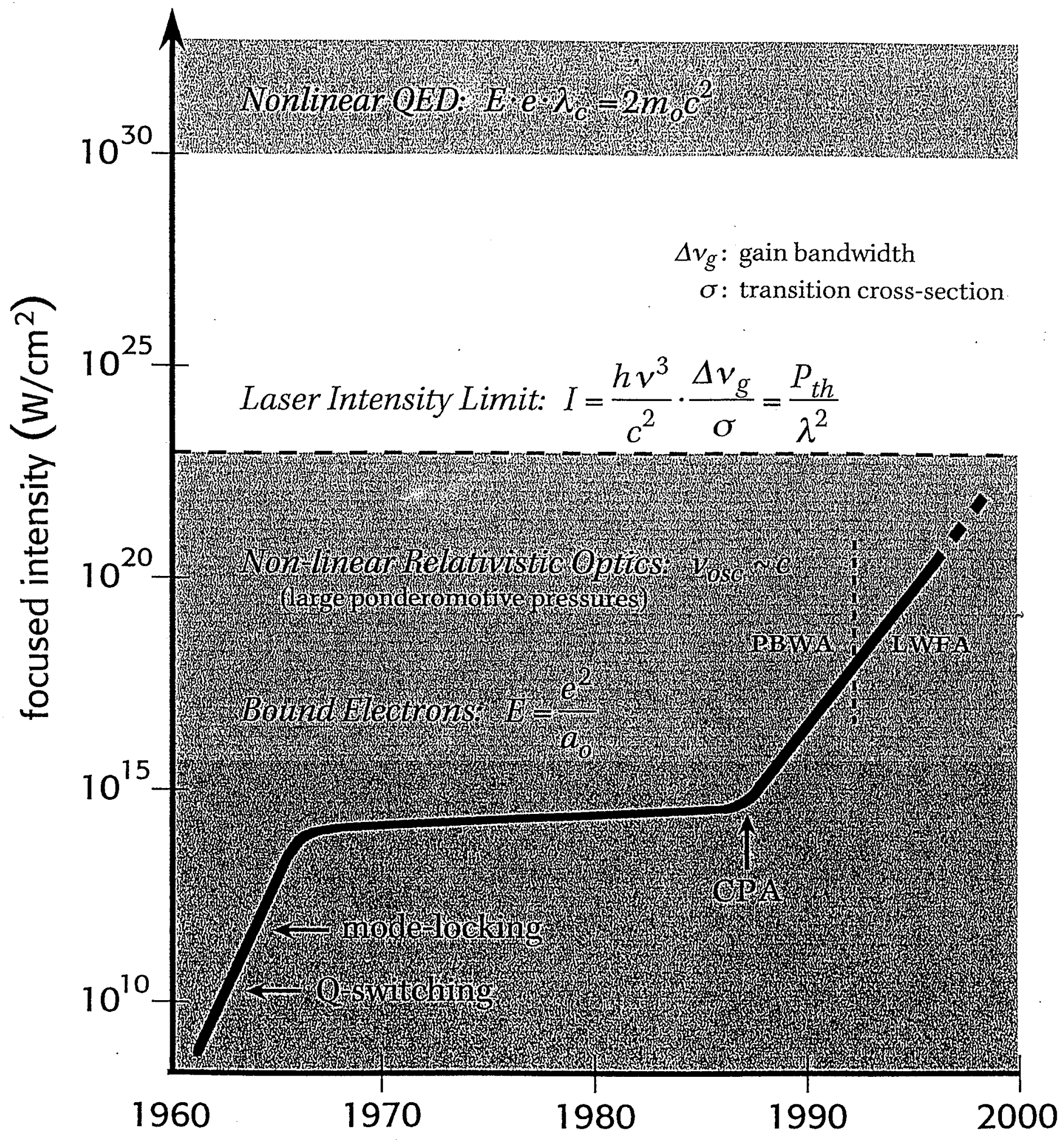




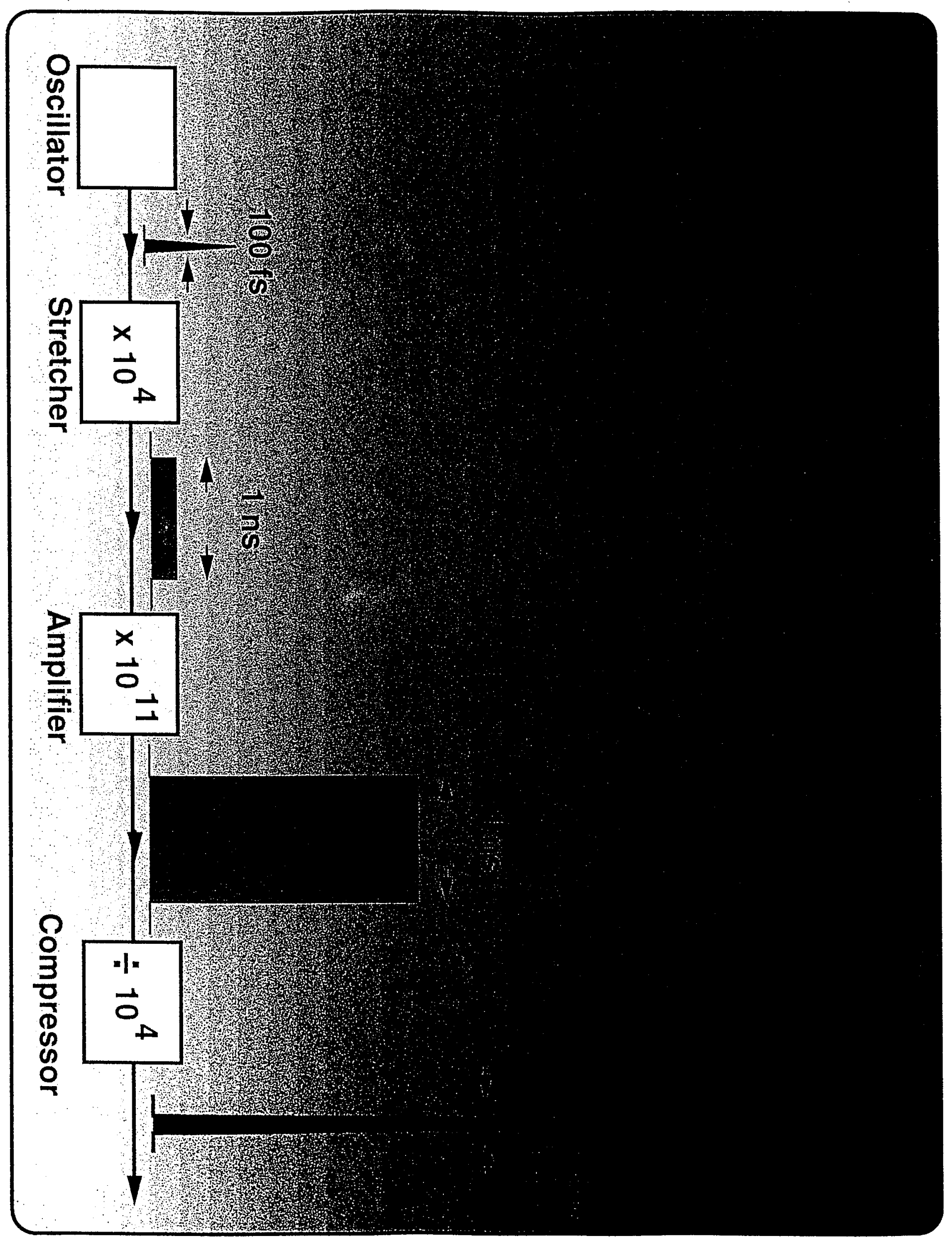




\title{
Ultrahigh-Intensity Laser: Physics of the Extreme on a Tabletop
}

\author{
Gérard A. Mourou, ${ }^{1}$ Christopher P. Barty, ${ }^{2}$ Michael D. Perry ${ }^{3}$ \\ ${ }^{1}$ Center for Ultrafast Optical Science, University of Michigan, 1006 I.S.T., 2200 Bonisteel Blvd., Ann Arbor, MI \\ 48109-2099; phone: 313-763-4875; fax: 313-763-4876 \\ ${ }^{2}$ Institute for Nonlinear Science, University of California, San Diego, Urey Hall Addition 3050 G, La Jolla, CA \\ 92093-0339; phone: 619-534-2417; fax: 619-534-7654 \\ ${ }^{3}$ Lawrence Livermore National Laboratory L 439, P.O. Box 808, Livermore, CA 94550; phone: 510-423-4915; fax: \\ 510-422-5537
}

\section{Introduction}

Advance in science is often triggered by technological breakthroughs. Over the past 10 years, 1 laser intensities have increased by more than four orders of magnitude to reach enormous intensities of $10^{20} \mathrm{~W} / \mathrm{cm}^{2}$ (Figure 1). The field strength at these intensities is of the order of a teravolt $/ \mathrm{cm}$, or 100 times the Coulombic field that binds the electrons to the nucleus. The electrons driven by such a field are relativistic, with an oscillatory energy of $10 \mathrm{MeV}$. At these intensities, the light pressure, $P=2 I / c$, is extreme, of the order of giga- to terabars. The laser interacting with matter-gas, solid, plasma-will generate high-order harmonics, energetic ions or electrons, gigagauss magnetic fields, and violent accelerations of $10^{20} g$, where $g$ is earth gravity. Finally, the interaction of an ultraintense beam with superrelativistic particles can produce fields greater than critical and nonlinear quantum electrodynamical effects can be observed. In many ways, this physical environment of extreme fields, pressure, magnetic field, temperature, and acceleration can only be found in stellar interiors or close to the horizon of a black hole and it is fascinating to think that an astrophysical environment governed by hydrodynamics, radiation transport, and gravitational interaction could be re-created in university laboratories for an extremely short time, switching the role of scientists from voyeurs to actors.

Also, ultrahigh-intensity lasers will play an important role in the development of new compact, tabletop-size, ultrashort sources of high-energy photons ( $x$ ray) and electrons (giga-electronvolt) with attosecond duration. The period of light being $3 \mathrm{fs}$ at $1 \mu \mathrm{m}$, getting into the attosecond regime will require shorter wavelengths, which naturally involves high intensities. For instance, short wavelengths can be produced by nonlinear effects like harmonic generation and produce subfemtosecond pulses. Similarly, the enormous field gradients that can be produced in laser plasma interaction can accelerate electrons over a distance $10^{4}$ times shorter than what was previously possible and the resulting electron pulses are expected to be well in the subfemtosecond range. These ultrafast sources will have important applications for time resolving the structure of matter at the atomic or molecular level. Short pulses are necessary, 
considering that the atomic motion is in the 100-fs range. Ultrahigh intensity led to a dramatic extension to the giga-electron-volt range of laser applications, until then confined to the $1-10-\mathrm{keV}$ regime.

What is spectacular is that the size of the laser involved is extremely compact (tabletop) and relatively inexpensive. It can deliver high average power and be built and operated by students.

Some of these tabletop-laser principles can be easily implemented at a minimum cost on existing large laser systems already built for laser fusion. Lawrence Livermore Laboratory, ${ }^{1} \mathrm{CEA}-$ Limeil, ${ }^{1}$ and Osaka ${ }^{1}$ have converted their nanosecond lasers to subpicosecond pulse duration, pushing their peak power by 3 orders of magnitude from the terawatt $\left(10^{12}\right)$ to $100-1000 \mathrm{TW} .1$ They also can be moved to very large structures like synchrotrons (ALS, ESRF) to produce ultrashort, high-energy $x$ rays by Compton scattering, or to accelerator facilities like SLAC to test nonlinear quantum electrodynamics by the interaction of the high-intensity pulses with superrelativistic electrons.

Figure 1 shows laser intensity as a function of years. We can see a rapid increase in intensity in the early $1960 \mathrm{~s}$, followed by a long plateau at $10^{15} \mathrm{~W} / \mathrm{cm}^{2}$. It took about 20 years, until 1987, for laser power to increase again. It is important to stress the similarity in slopes between the early 1960s and the past ten years, and to remember that it is during this period of very rapid increase in intensities in the 1960s that most of the nonlinear optics phenomena were discovered. In a similar way, the spectacular increase in intensity (four to five orders of magnitude) that we are experiencing today should lead tó exceptional scientific discoveries. Finally, from an educational point of view, this compact laser offers the advantage of bringing some of the research traditionally done on large instruments to human-size setups in university laboratories. Their small scale and large numbers should greatly foster diversification and multidisciplinarity and should attract students to scientific disciplines.

\section{The Evolution of Laser Peak Power}

Although strong-field physics with conventional lasers had already started in the $1960 \mathrm{~s}^{2}$ the scientific revolution started about 10 years ago. At that time, we demonstrated that ultrashort pulses at the 100-fs level could be amplified, without damaging the amplifying media, to the terawatt level, using a technique that we called Chirped Pulse Amplification (CPA). ${ }^{3}$

Since their inception in 1960, the peak power of lasers has evolved by a succession of leaps of three orders of magnitude. They were produced each time by decreasing the pulse duration accordingly. First the lasers were free running, with a duration in the $10-\mu$ s and peak power in the kilowatt range. In 1962, modulation of the laser-cavity quality factor allowed the same 
energy to be released in a nanosecond time scale, a thousand times shorter, to produce pulses in the megawatt range. In 1964, locking the longitudinal modes of the laser (mode locking) allowed the laser pulse duration to be reduced by another factor of a thousand, down to the picosecond level, pushing the peak power a thousand times higher, to the gigawatt level. At this point, the intensities associated with the ultrashort pulses were becoming prohibitively high-i.e., in the $\mathrm{GW} / \mathrm{cm}^{2}$ range. At these intensities, the index of refraction becomes linearly dependent on the intensity to vary like $n=n_{0}+n_{2} I$, where $n$ is the index of refraction, $n_{0}$ the index of refraction at low intensity, $n_{2}$ the nonlinear index of refraction, and $I$ the intensity. The result is that, for a beam with a Gaussian radial intensity distribution, the center of the beam sees a larger index of refraction than its sides. The material becomes a positive lens and alters the beam wave-front quality to an unacceptable level, to the point where it can create filaments and irreversible damage to the laser system. The only way to increase the peak power was to increase the beam diameter at the expense of size, low repetition rate, and cost.

Although the pulse duration kept decreasing, for about 20 years the intensity limitation in laser systems kept the peak power about constant at the gigawatt level for a $\mathrm{cm}^{2}$ beam, until 198587, when the technique of CPA was demonstrated. The CPA concept is illustrated in Fig. 2 . The short pulse is first produced by an oscillator. After generation it is not amplified directly, but stretched by a large amount, $10^{3}$ to $10^{5}$, from femtosecond to nanosecond, reducing the intensity accordingly. The pulse intensity is now low enough that the stored energy can be safely extracted out of the amplifier, without fear of beam distortions and damage. Once the stored energy is fully extracted, the pulse is recompressed, ideally to its initial value. The key point of the CPA technique is that it decouples pulse fluence (energy $/ \mathrm{cm}^{2}$ ) and pulse intensity (power $/ \mathrm{cm}^{2}$ ). So it reconciles two apparently conflicting needs: to have (a) the highest fluence for efficient energy extraction and (b) minimum intensity to avoid the undesired nonlinear effects.

CPA had a dramatic impact. First, we could for the first time use extremely good energy storage media like Nd:glass, ${ }^{3}$ alexandrite, ${ }^{1}$ Ti:sapphire, ${ }^{1}$ and $\mathrm{Cr}: \mathrm{LiSAF}^{1}{ }^{1}$ and extract their energy fully. Before CPA, dyes and excimers, which are a thousand times inferior in energy storage, were the only choice for ultrashort pulse generation. So at equal size, a CPA laser system, using the superior energy storage media, could produce $10^{3}$-to- $10^{4}$ times higher peak power than conventional dye or excimer systems. Second, CPA could be easily adapted on very large-scale, expensive lasers already built for laser fusion. By simple beam manipulations, a stretching at the beginning and a compression at the end of the amplifying chain, these expensive systems built to amplify nanosecond pulses to the terawatt level could be converted to amplify femtosecond pulses, as well, and to produce petawatt pulses. 
It is interesting to note that this sudden improvement by three-to-four orders of magnitude is similar to the one in electronics in 1960-when they went from discrete $(\mathrm{mm})$ to integrated $(\mu \mathrm{m})$ circuits; or to the advent of laser diodes-where meter-size lasers could be miniaturized to millimeters. As in electronics, this enormous reduction in size led to new advantages: speed, efficiency, reliability, and cost.

\section{Unleashing Laser Power: Chirped Pulse Amplification (CPA)}

CPA (Fig. 2) involves impressive manipulations. Stretching by $10^{3}-10^{5}$, amplification by $10^{11}$ (nanojoules to tens of joules), and recompression by $10^{3}$ to $10^{5}$. Because we want the highest intensities on target, it is important that these manipulations be performed with the highest degree of fidelity, so that the pulse in the time and spatial domains possesses the highest temporal contrast and most of the energy will be in the diffraction-limited spot. Two main hurdles had to be overcome for the generation of ultrahigh peak power: (a) the accommodation of the large stretching-compression ratio and (b) the amplification of large pulse spectra.

\section{a. Clearing the First Hurdle: Discovery of the Matched Stretcher}

We may wonder how we can stretch and compress a short pulse over so many orders of magnitude with impunity and fidelity. This came with the discovery of the matched stretchercompressor. The first CPA system ${ }^{3}$ was using the positive group velocity dispersion of a singlemode fiber. At that time, the only practical way we knew, to stretch a pulse was with positive group velocity dispersion (where the red wavelength goes faster than the blue). Once it had been amplified, the only way we knew to compress the pulse was to use a pair of parallel gratings. Such a pair exhibits a negative group velocity dispersion, as demonstrated by Treacy in $1969^{1}$ (blue goes faster than the red, Fig. 2a), so the pulse could be put back together. However, the stretcher and compressor did not have their dispersive characteristics perfectly matched, so the recompression was not perfect, leading to temporal wings in the pulse, hence limiting the stretching-compression ratio to about 100 .

We became aware of a "compressor" designed by O. Martinez, ${ }^{4}$ built for optical communication to compress pulses at $1500 \mathrm{~nm}$. We recall that in this spectral range, light experiences a negative group velocity dispersion. This new dispersive system was composed of a telescope of magnification of one between two antiparallel gratings (Fig. 2b). Studying this system, it became clear to us that it had the exact same dispersive function as the Treacy compressor, but with the opposite sign. In other words, it was the perfect conjugate of the Treacy compressor-with the important consequence that any arbitrary short pulse could in theory be stretched to any pulse duration and put back to its original shape. A stretching factor of $10^{3}-10^{4}$ was immediately demonstrated with a Nd:glass system, to produce the first tabletop terawatt laser system. ${ }^{3}$ 
With the discovery of the matched stretcher-compressor, a very high hurdle was cleared. For the amplification of pulses at the 10-fs level, stretching up to $10^{5}$ can be required ${ }^{5}$ In this case, it is necessary to design the expander not to match the compressor alone but instead to match the sum of the phase function of the gain medium, mirrors, and other optical elements in the amplification chain. The other alternative, used by Kapteyn et al., 5 is to use very short-length amplifying media and to minimize the stretching ratio. Due to the very high peak power, the pulse after compression must be in vacuum to avoid nonlinear effects in air ${ }^{14}$ that will produce wave-front distortion and filamentation.

\section{b. Clearing the Second Hurdle: The Discovery of Ultrabroadband Gain Media}

Ultrashort pulses have enormous spectra. From the uncertainty principle $\Delta v \cdot \tau=0.35$, where $\Delta v$ is the pulse spectrum and $\tau$ is the pulse duration, a 10 -fs pulse will have a bandwidth of $70 \mathrm{~nm}$. All the spectral components will have to be equally amplified over many orders of magnitude at the risk of narrowing its bandwidth and lengthening its duration. For ultrashort pulses, this limitation was removed with the invention of ultrabroadband amplification media, such as Ti:sapphire ${ }^{6}$ and Cr:LiSAF, 7 and with the development of techniques such as the regenerative pulse shaping introduced by Barty et al..$^{5}$ to eliminate gain narrowing during the amplification process. In particular, Ti:sapphire, whose gain bandwidth can theoretically support the amplification of pulses of less than $5 \mathrm{fs}$ in duration, is ideally suited for CPA because of its excellent thermal and optical properties and moderate saturation fluence. Because of the excellent thermal properties, ultrashort pulses could be amplified at a much larger repetition rate, $10-10^{3} \mathrm{~Hz}$, bringing the average power of Ti:sapphire-based CPA to the watt level. 8,5 This represents an improvement of 2-3 orders of magnitude over conventional amplification systems based on dyes or excimers. Increased repetition rate at constant peak power leads directly to higher experimental utility. With ultrafast CPA systems, for instance, it is possible to apply signal-averaging techniques to high-field laser/matter investigations.

At the University of California, San Diego, 4-TW, sub-20-fs FWHM pulses ${ }^{5}$ have been demonstrated from a Ti:sapphire amplification chain operating at a $10-\mathrm{Hz}$ repetition rate. This laser is shown in Fig. 4. At ENSTA, France, the University of Tokyo, and the Japanese Atomic Energy Research Institute, ${ }^{5}$ 30-TW, 20-30-fs pulses ${ }^{5}$ have been obtained. Extension of these concepts to higher peak power and higher repetition rate are straightforward and are currently under development. In Japan, the Kansai research establishment of the Japanese Atomic Energy Research Institute is presently designing and testing components for a petawatt (20-J, 20-fs), 1-Hz laser which is scheduled for construction in 1999. In Bordeaux, France, and at the NSF Center for Ultrafast Optical Science (CUOS) at the University of Michigan, funds have been 
allocated and designs completed for the construction a 1-TW (20-mJ, 20-fs), 1-kHz laser facilities.

\section{The Generation of Ultrashort Pulses and Their Characterization}

Ultrashort pulses as short as a few optical cycles have been with us for 10 years. But it is the outstanding discovery by W. Sibbett in 1991 of Kerr Lens Mode Locking (KLM) ${ }^{9}$ with its successive refinements ${ }^{11}$ that made the generation of pulses shorter than 10 fs routine. These systems will provide a robust source of short seed pulses at the nanojoule level for CPA systems.

The concept of the generation of ultrashort pulses by KLM is simple and uses simultaneously the very large bandwidth of Ti:sapphire and its optical Kerr effect, or the intensity-dependent index of refraction, described above. A simplified diagram of a laser cavity is shown in Fig. $3 a$. The cavity is composed of curved mirrors and the amplifying medium (Ti:sapphire). The amplifier is optically pumped, usually with a continuous-wave argon laser. The Ti:sapphire has a double role. The first is to amplify the pulse, to compensate the various losses in the cavity. The second is to be a lens with an intensity-dependent focal length. We have seen previously that the Gaussian beam profile will produce a positive lens due to the intensity-dependent index of refraction. So the experimentalist adjusts the wave-front radius of curvature to be equal to one of the mirrors for the highest intensity or the shortest pulse duration. In other words, it is where the laser experiences the minimum amount of losses that the laser will run. To get to the ultimate pulse duration, the cavity mirrors (no lenses to avoid dispersive effects) must be broadband enough and the dispersion of the pulse in crystal must be compensated 10 by frequency-chirped mirrors (Krausz and coworkers) or by a sequence of prisms (Murnane and Kapteyn group). Pulses down to $6.5 \mathrm{fs}$ have been produced directly out of the oscillator by U. Keller et al. 10

\section{The Petawatt: The Heavyweight CPA}

The Petawatt Project, to explore the feasibility of the fast ignition concept, was realized at Livermore by one of the authors. It illustrates beautifully the adaptability of CPA. They use a beam of the large NOVA, a Nd:glass laser chain built for nanosecond pulse amplification delivering kilojoules in a few nanoseconds. Although conceptually simple, the adaptation of CPA on this laser chain required the development of sophisticated new technology: in particular, the design of very large diffraction gratings with $75-\mathrm{cm}$ diameter, efficiency greater than $90 \%$, with flatness-better than $\lambda / 10$, and a good damage threshold. Thus, $1.3 \mathrm{~kJ}$ could be extracted in $800 \mathrm{ps}$ and compressed in $430 \mathrm{fs}$, producing $1.3 \mathrm{PW}$ with $>10^{21} \mathrm{~W} / \mathrm{cm}^{2}$ 
irradiance 11 - the highest peak power and irradiance ever produced. Currently, the petawatt operates with a $46-\mathrm{cm}$ beam and $75-\mathrm{cm}$ grating. These large gratings forming the compressor were mounted in a vacuum chamber to avoid self-focusing in air. ${ }^{14}$ These gratings were increased to the full $94-\mathrm{cm}$ size in July 1997, thereby allowing operation of the petawatt at $1 \mathrm{~kJ}$ with a $58-\mathrm{cm}$ diameter beam.

\section{How High Can the Peak Power Go?}

Is it possible to predict the ultimate peak power that we will able to achieve for a given-size system? A good estimate can be obtained using the following simple argument. The maximum energy that can be extracted from an amplifier is of the order of the saturation fluence, $F_{s}=\frac{h v}{\sigma}$, where $\sigma$ is the transition cross section, while the shortest pulse duration $\tau$ is limited by the gain bandwidth of the amplifying medium, $\Delta v$. The maximum theoretical peak power per $\mathrm{cm}^{2}$ of beam is therefore given simply by $P_{t h}=\frac{h v \Delta v}{\sigma}$. It is, in fact, the intensity necessary to flip the population in a time corresponding to the dephasing time of the transition. This quantity is known in quantum electronics as the Rabi intensity. The maximum focusable intensity will then be equal to this power over a spot size corresponding to $\lambda^{2}$. The highest intensity will therefore be $I_{t h}=\frac{h v^{3} \Delta v}{c^{2} \sigma}$. The highest peak power will be produced by amplifying media with the smallest transition cross section and the largest bandwidth.

Here are some examples. $P_{\text {th }}$ varies from 200 TW for Ti:sapphire to 3000 TW for $\mathrm{Yb}$ :glass. Using Yb:glass, a material that can be obtained in large dimensions, a system with a beam size of $10 \mathrm{~cm}$ by $10 \mathrm{~cm}$ could produce peak power of $0.3 \mathrm{EW}$ (an exawatt is $10^{18} \mathrm{~W}$ ). This power, focused over a diffraction-limited spot size of a few $\mu \mathrm{m}^{2}$, could produce on-target intensities in the $10^{24} / \mathrm{cm}^{2}$ range!

\section{Ultrahigh-Intensity Applications}

Figure 1 shows the laser intensity and the different physical regimes that are becoming accessible. Because of the intense activities that this field experiences, it is impossible in an exhaustive manner to do justice to all contributors. We will stress in the following only some of the most recent works and directions that occurred over the past few years.

\section{At $10^{14} \mathrm{~W} / \mathrm{cm}^{2}$}

High harmonic generation from gases ${ }^{2}$ is still a fascinating and active topic. The bound electrons are driven by the high field. Their response is highly nonlinear and harmonics of the laser to very large orders are emitted. These harmonics can be generated up to a cutoff frequency ${ }^{2}$ given by 
$h v_{n}=E_{i}+3.2 U_{p}$, where $h$ is the Planck constant, $v_{n}$ the harmonic frequency, $E_{i}$ the ionization energy, and $U_{p}$ the quiver energy. It was recently found that by shortening the pulse to a few optical periods, $10-20 \mathrm{fs}$, higher ionization energy could be obtained, ${ }^{12}$ pushing the harmonic number over 300 -that is, well within the water window. ${ }^{13}$ An interesting aspect of high harmonic generation is that it may provide a way to generate pulses of attosecond duration. At these intensities, when the main pulse freely propagates, due to the combination of diffraction, intensity-dependent index of refraction $\left(n_{2}\right)$, and field ionization, it can condense into "light bullets $^{\prime \prime 14}$ of $80-\mu \mathrm{m}$ diameter that can propagate over distances greater than $100 \mathrm{~m}$. Important applications of this channeling are currently being investigated: guiding for efficient harmonic generation in gases and electron acceleration, and control of lightning for airport and nuclear plant protection as proposed by J. C. Diels. ${ }^{14}$ In solids at $10^{15} \mathrm{~W} / \mathrm{cm}^{2}$, a plasma forms, light is absorbed, and material damaged. First, the free electrons are generated by single- or multiphoton absorption. They are then multiplied to reach the critical density where the plasma can absorb the laser energy, due to the great number of collisions $\left(10^{15} \mathrm{~Hz}\right)$, to finally damage the material. Because the single- or multiphoton absorption is a deterministic ${ }^{15,16}$ process and the impact ionization as a function of the field saturates, ablation with ultrashort pulses becomes very deterministic (accurate threshold)-as opposed to stochastic for long pulses. This deterministic character may lead to some of the most important practical applications of ultrashort pulses: eye surgery, micromachining, and data storage. 16

Intensities $10^{16} \mathrm{~W} / \mathrm{cm}^{2}$

Laser interaction with clusters is becoming very popular and shows enhanced harmonic generation and $x$-ray production, as well as a copious amount of high-energy electrons up to $3 \mathrm{keV}$ and ions up to $1 \mathrm{MeV} .{ }^{17}$ In solids, 18 the combination of high electron temperature and large ponderomotive pressure, which at these intensities become comparable to the thermal pressure (see the work of Umstadter et al. ${ }^{2}$ ), contribute to increase the mean free path. As a result, collisional absorption is suppressed to give rise to collisionless absorption like resonance absorption or by anomalous skin effect, when the collision mean free path becomes larger than the skin depth.

The electrons driven through steep gradients can radiate very high harmonics. Up to the 70 th harmonic was produced with a 2.5-ps CPA laser. ${ }^{18}$ Harmonic generation in solids is very sensitive to prepulse, which affects the gradient steepness. Very high harmonics, order $n>100$, should be produced with good efficiency $\left(>10^{-5}\right)$ with shorter $(100-\mathrm{fs})$ and cleaner pulses (see Von der Linde ${ }^{19}$ ). 


\section{Intensities $>10^{18} \mathrm{~W} / \mathrm{cm}^{2}$}

At these intensities, the electrons in the field of the laser oscillate with a quiver velocity near the speed of light (quiver energy becomes equal to the rest mass, i.e., $0.5 \mathrm{MeV}$ ), and mass increases by the relativistic factor $\gamma$. For light at $1 \mu \mathrm{m}, \gamma$ equals $\sqrt{2}$. The force applied to the electron is expressed by the Lorentz equation $F=q \cdot\left(E+\frac{v}{c} \wedge B\right)$, where $q$ is the charge of the electron, $E$ and $B$ the electric and magnetic fields) of the laser, $v$ the quiver velocity, and $c$ the speed of light. In conventional linear or nonlinear optics, the force due to the magnetic field is always neglected due to the small quiver velocity of the electron compared to $c$. However, at $10^{18} \mathrm{~W} / \mathrm{cm}^{2}, v$ is close to $c$, and the magnetic and electric force become equal, opening up the new field of relativistic nonlinear optics. This regime is characterized by extremely large-greater than gigabar-light pressure, much larger than the thermal pressure. Because the laser is focused on a very small spot size of $10 \mu \mathrm{m}$ (several wavelengths), a large lateral gradient will be produced that will have the tendency to push the electrons from the center of the focal spot, dragging the more massive ions with them. 20 The beam center will be evacuated, cavitation 20 decreasing both ion and electron densities. The simultaneous decrease of the electron density and plasma frequency $\omega_{p}=\frac{\omega_{p o}}{\gamma}$, where $\omega_{p o}$ is the plasma frequency without mass correction, will lead to induced transparency. That may have been observed recently by Giuletti et al. ${ }^{19}$

In subcritical gases, this enormous ponderomotive pressure produced by a focused pulse can displace the electrons in its way. The electrons will be repelled by the ions once the pulse goes by, 21 producing a large plasma wave with electrostatic fields greater than $200 \mathrm{GeV} / \mathrm{m}$ or $10^{4}$ larger than what can be produced with conventional technologies. This concept proposed by Tajima and Dawson is known as laser wakefield acceleration. ${ }^{21}$ Collimated electron beams of a few nanocoulombs with mega-electron-volt energy have been produced by this simple technique, ${ }^{22}$ where the laser pulse is focused onto a gas jet $1 \mathrm{~mm}$ thick (Fig. 5). A scheme to inject electrons in the narrow acceleration bucket of about 50 fs has been proposed by Umstadter. ${ }^{22}$ Because the electrons become immediately relativistic, the space charge broadening will be minimum. According to simulations, subfemtosecond pulses of electrons could be produced. At these intensities, the plasma frequency, which is intensity-dependent due to the mass change, will vary across the laser spot size, altering the index of refraction accordingly, causing the laser to self-focus to a $\mu \mathrm{m}$-size filament. ${ }^{21}$ This is known as relativistic self-focusing, predicted by Litvack and Max ${ }^{21}$ in the 1970s. A large current density, of the order of $10^{12} \mathrm{~A} / \mathrm{cm}^{2}$, will circulate in the filament to produce an extremely large magnetic field of gigagauss strength. 20 
In wakefield acceleration, electrons are accelerated from a cold start to $c$ in a fraction of a picosecond corresponding to $10^{21} \mathrm{~g}$ that could lead to detectable Unruh radiation. ${ }^{23} \mathrm{~A}$ recent experiment on solid targets ${ }^{19}$ by Saubrey et al. has already confirmed plasma acceleration of $10^{17} \mathrm{~g}$ with a modest intensity by today's standard of $10^{18} \mathrm{~W} / \mathrm{cm}^{2}$. This kind of acceleration is the one found near the horizon of a black hole. This result illustrates the exciting possibility for ultrahigh intensity to test general relativity in a laboratory setting.

In solids, one of the most impressive applications is the so-called Fast Ignitor. ${ }^{1}$ The essential idea, as illustrated in Fig. 6, is to pre-implode a deuterium-tritium capsule to an isochoric (uniform-density) condition (see J. C. Kieffer et al. ${ }^{1}$ ). At the point of maximum compression, irradiate the side of the imploded core with a laser pulse much shorter than the hydrodynamic disassembly time of the irradiated spot $\left(\tau \approx \frac{R_{\text {spot }}}{v_{s}} \leq 10 \mathrm{ps}\right)$. Hot electrons $(200 \mathrm{keV}<E<1$ $\mathrm{MeV})$ generated by the interaction of the intense $\left(10^{19}-10^{21} \mathrm{~W} / \mathrm{cm}^{2}\right)$ light with plasma rapidly equilibrate in the dense fuel. The energy equilibration of the electrons raises the overall ion temperature to $5-20 \mathrm{keV}$, initiating fusion burn. High laser pulse energy is required in order to produce enough hot electrons to heat a sufficient number of ions-to initiate fusion burn. In addition, nearly perfect beam quality is required in the laser in order to achieve a small spark region. The Fast Ignitor concept offers the possibility of high target gain at reduced total drive energy, compared with conventional ICF.

It has been suggested by Tajima, Takabe, Ichimaru, and Remington ${ }^{19}$ that astrophysical conditions such as opacity, densities, and temperatures could be reproduced and tested at intensities of $10^{20} \mathrm{~W} / \mathrm{cm}^{2}$, corresponding to gigabar pressures. One proposal is to look at the rates of nuclear fusion, such as thermonuclear and pycnonuclear, that are greatly modified by the state of dense matter. Ultrahigh-pressure metal physics with ultrahigh-intensity lasers could be applied to understand the phase transformation, metallization, and crystallization of hydrogen in stellar interiors and help the investigation of the physical mechanisms governing supernovae, stars, and nebulae.

\section{Nonlinear quantum electrodynamics}

The possibility of creating electron-positron pairs from the laser field was proposed in the late 1960s. As seen in Fig. 1, pair creation directly from the laser will require intensity of the order of $10^{30} \mathrm{~W} / \mathrm{cm}^{2}$. With $E$ as the laser field, it is the energy required over the Compton wavelength, $\lambda_{c}=\frac{h}{m_{0} c}$, to create an electron-positron pair. The field is therefore equal to $E=\frac{2 m_{0} c^{2}}{e \lambda_{c}}$ and corresponds to $10^{16} \mathrm{~V} / \mathrm{cm}$, which is about four-to-five orders of magnitude above the laser field of today's laser. This enormous gap was bridged 24 by using the electric field enhancement 
produced in the frame of superrelativistic electrons. Using the $50-\mathrm{GeV}$ electron beam at the Stanford Linear Accelerator, corresponding to a $\gamma$ of $10^{5}$, with a currently available high-power laser, the field is enhanced to $E \sim 10^{16} \mathrm{~V} / \mathrm{cm}$, exceeding the critical field. The multiphoton pair production, $w_{\gamma}+n w_{0} \rightarrow e^{+}+e^{-}$, has been observed, where $w_{\gamma}$ is the upconverted frequency from Compton Scattering and $w_{0}$ is the laser frequency. The same group is studying nonlinear Compton scattering, $e+n w_{0} \rightarrow e^{\prime}+w_{\gamma}$. In this case, the high-energy $\gamma$ ray produced in the laser focus by an incident electron interacts before leaving the focal region. Up to the $n=4$ process has been observed.

Work cited above was supported by the National Science Foundation through the Center for Ultrafast Optical Science under STC PHY 8920108; by the U.S. Department of Energy through University of California Lawrence Livermore National Laboratory DOE contract W-7405-ENG-48; and by the University of California Los Alamos National Laboratory GB/020165/AL/03. 


\section{Figure Captions}

1. Laser intensity vs. years showing the different physical thresholds.

2 (a) The CPA concept, whereby an ultra short pulse is stretched by a large factor, $10^{3}-10^{5}$, amplified by $10^{11}$, and recompressed by $10^{3}-10^{5}$.

(b) The Matched Stretcher-Compressor. The stretcher is composed of a pair of telescopes of magnification of one between two anti-parallel gratings. Note that in this configuration the red wavelengths have a shorter path than the blue ones. Conversely the compressor is composed of a pair of parallel gratings where the optical length for the blue is shorter than for the red.

3 (a) A schematic of an ultrashort pulse cavity. The use of chirped mirrors makes the cavity dispersion free, in order to accommodate the very large Fourier spectrum of a sub-10femtosecond pulse.

(b) The autocorrelation trace of the output pulse produced by mixing the signal with itself in a second harmonic crystal.

4. A schematic of the 50-TW and 5-TW Ti:sapphire CPA systems (Barty et al.) ${ }^{5}$

5. The impact of a high-energy, collimated beam of electrons (up to $50 \mathrm{MeV}$ ) on a phosphor screen produced by the interaction of the ultraintense pulse with a 1-mm gas jet of He. The laser is blocked by an Al foil.

6. Diagram of fast ignition concept in inertial confinement fusion. 


\section{References}

1. M. D. Perry and G. Mourou, Science 264, 917 (1994).

2. C. Joshi and P. B. Corkum, Physics Today 48, 36-43 (1995).

3. D. Strickland and G. Mourou, Opt. Commun. 56, 219 (1985); P. Maine, D. Strickland, P. Bado, M. Pessot, and G. Mourou, IEEE J. Quantum Electron. 24, 398 (1988).

4. O. E. Martinez, ibid. 23, 1385 (1987).

5. B. E. Lemoff and C. P. Barty, Opt. Lett. 18, 1651 (1993); J. Zhou, G. Taft, C. P. Huang, M. M. Murnane, H. C. Kapteyn, I. Christov, ibid. 19, 1149 (1994); C. Barty, T. Guo, C. LeBlanc, F. Raski, C. Rose-Petruck, J. Squier, K. Wilson, V. Yakolev, and K. Yamakawa, ibid. 20, 668 (1996); J. P. Chambaret, C. LeBlanc, G. Cheriaux, P. Curley, G. Darpentigny, and P. Rousseau, ibid. 21, 1921 (1996); Japan Atomic Energy Research Institute, private communication, Watanabe, University of Tokyo; K. Yamakawa, M. Aoyama, S. Matsuoka, Y. Akahane, T. Takuma, C. P. Barty, and D. Fittinghoff, "International Conference on Superstrong Fields in Plasmas," Varenna, Italy (1997); J. Itatani, Y. Nabekawa, K. Kondo, and S. Watanabe, Opt. Commun. 134, 134 (1995).

6. P. F. Moulton, J. Opt. Soc. Am. B 3, 125 (1986).

7. S. A. Payne, L.L.Chase, L. K. Smith, W. L. Kway, and H. W. Newkirk, J. Appl. Phys. 66, 1051 (1989).

8. F. Salin, J. Squier, G. Mourou, G. Vaillancourt, Opt. Lett. 16, 1964 (1991); T. B. Norris, ibid. 17, 1009 (1992); S. Backus, C. Durfee, G. Mourou, H. C. Kapteyn, M. Murnane, Opt. Lett. 22, 1256 (1997).

9. C. V. Shank, Ultrashort Laser Pulses and Applications, ed. by W. Kaiser (Springer-Verlag, Berlin, 1988), Vol. 60, pp. 5-34; D. E. Spence, P. N. Kean, W. Sibbett, ibid. 16, 42 (1991).

10. J. Zhou, G. Taft, C. P. Huang, M. M. Murnane, H. C. Kapteyn, I. Christov, ibid. 19, 1149 (1994); Ch. Spielmann, P. F. Curley, T. Brabec, F. Krausz, IEEE J. Quantum Electron. 30, 1100 (1994); A. Stingl, Ch. Spielmann, F. Krausz, R. Szipöcs, Opt. Lett. 19, 204 (1994); I. D. Jung, F. X. Kärtner, N. Matuschek, D. H. Sutter, F. Morier-Genoud, G. Zhang, U. Keller, V. Scheuer, M. Tilsch, and T. Tschudi, ibid. 22, 1009 (1997).

11. M. D. Perry, B. C. Stuart, G. Tietbohl, J. Miller, J. A. Britten, R. Boyd, M. Everett, S. Herman, H. Nguyen, H. T. Powell, and B. W. Shore, CLEO'96 Technical Digest Series (Opt. Soc. America, Washington, DC, 1996), Vol. 9, pp. 307-308.

12. J. Zhou, J. Peatross, M. Murnane, H. Kapteyn, I. Christov, Phys. Rev. Lett. 76, 752 (1996). 
13. Krauss, CLEO 97; Z. Chang, A. Rundquist, H. Wang, H. Kapteyn, M. Murnane, to be published in Physical Review Letters (1997).

14. A. Braun, G. Korn, X. Liu, D. Du, J. Squier, and G. Mourou, Ultrafast Phenomena IX (Springer, Berlin, 1994), Vol. 60, p. 248.

15. D. Du, X. Liu, G. Korn, J. Squier, and G. Mourou, Appl. Phys. Lett. 64, 3071 (1994).

16. B. C. Stuart, M. D. Feit, A. M. Rubenchik, B. W. Shore, and M. D. Perry, Phys. Rev. Lett. 74, 2248 (1995); E. N. Glezer, M. Milosavljevic, L. Huang, R. J. Finlay, T.-H. Her, T. P. Callan, and E. Mazur, Opt. Lett. 21, 2023 (1996).

17. T. Ditmire, T. Donnelly, R. W. Falcone, and M. D. Perry, Phys. Rev. Lett. 75, 3122 (1995); T. Ditmire, J. W. G. Tisch, E. Springate, M. B. Mason, N. Hay, R. A. Smith, J. P. Marangos, and M. H. R. Hutchinson, Applications of High Field and Short Wavelength Sources VII (Opt. Soc. America, Washington, DC, 1997).

18. See, for instance, P. Gibbon and E. Förster, Plasma Phys. Control Fusion 38, 769 (1996).

19. D. von der Linde, "Generation of Ultrashort XUV Pulses by High-Order Harmonic Generation from Plasma-Vacuum Bound," "International Conference on Superstrong Fields in Plasmas," Varenna, Italy (1997); R. Sauerbrey, "Femtosecond Laser Plasmas, FROGS, and Black Holes," "International Conference on Superstrong Fields in Plasmas," Varenna, Italy (1997); A. Giulietti, "High-Intensity 30-fs Laser Pulse Interaction with Thin Foils," "International Conference on Superstrong Fields in Plasmas," Varenna, Italy (1997); H. Takabe, "Laboratory Astrophysics Using Intense and Ultraintense Lasers," "International Conference on Superstrong Fields in Plasmas," Varenna, Italy (1997); B. Remington, "Bringing the Stars Down to Earth with Lasers," "International Conference on Superstrong Fields in Plasmas," Varenna, Italy (1997); S. Ichimaru, "Ultradense Hydrogen: Frontiers in Astrophysics, Ultrahigh-Pressure Metal Physics, and Fusion Studies," "International Conference on Superstrong Fields in Plasmas," Varenna, Italy (1997).

20. A. Pukhov and J. Meyer-ter-Vehn, Phys. Rev. Lett. 7 6, 3975 (1996).

21. E. Esarey, P. Sprangle, J. Krall, and A. Ting, IEEE Trans. Plasma Sci. 24, 252 (1996).

22. A. Modena, Z. Najmudin, A. E. Dangor, C. E. Clayton, K. A. Marsh, C. Joshi, V. Malka, C. B. Darrow, C. Danson, D. Neely, and F. N. Walsh, Nature 377, 606 (1995); K. Nakajima, D. Fisher, T. Kawakubo, H. Nakanishi, A. Ogata, Y. Kato, Y. Kitagawa, R. Kodama, K. Mima, H. Shiraga, K. Suzuki, K. Yamakawa, T. Zhang, Y. Sakawa, T. Shoji, Y. Nishida, N. Yugami, M. Downer, and T. Tajima, Phys. Rev. Lett. 74, 4428 (1995); D. 
Umstadter, S.-Y. Chen, A. Maksimchuk, G. Mourou, and R. Wagner, Science 273, 472

(1996); S. Le Blanc, M. Downer, R. Wagner, S.-Y. Chen, A. Maksimchuk, G. Mourou, and D. Umstadter, Phys. Rev. Lett. 77, 5381 (1996).

23. P. Chin, and T. Tajima, to be published, Physical Review Letters.

24. C. Bula, K. T. McDonald, E. J. Prebys, C. Bamber, S. Boege, T. Kotseroglou, A. C. Melissinos, D. D. Meyerhofer, W. Ragg, D. L. Burke, R. C. Field, G. Horton-Smith, A. C. Odian, J. E. Spencer, D. Waltz, S. C. Berridge, W. M. Bugg, K. Shmakov, and W. Weidemann, Phys. Rev. Lett. 76, 3116 (1996). 


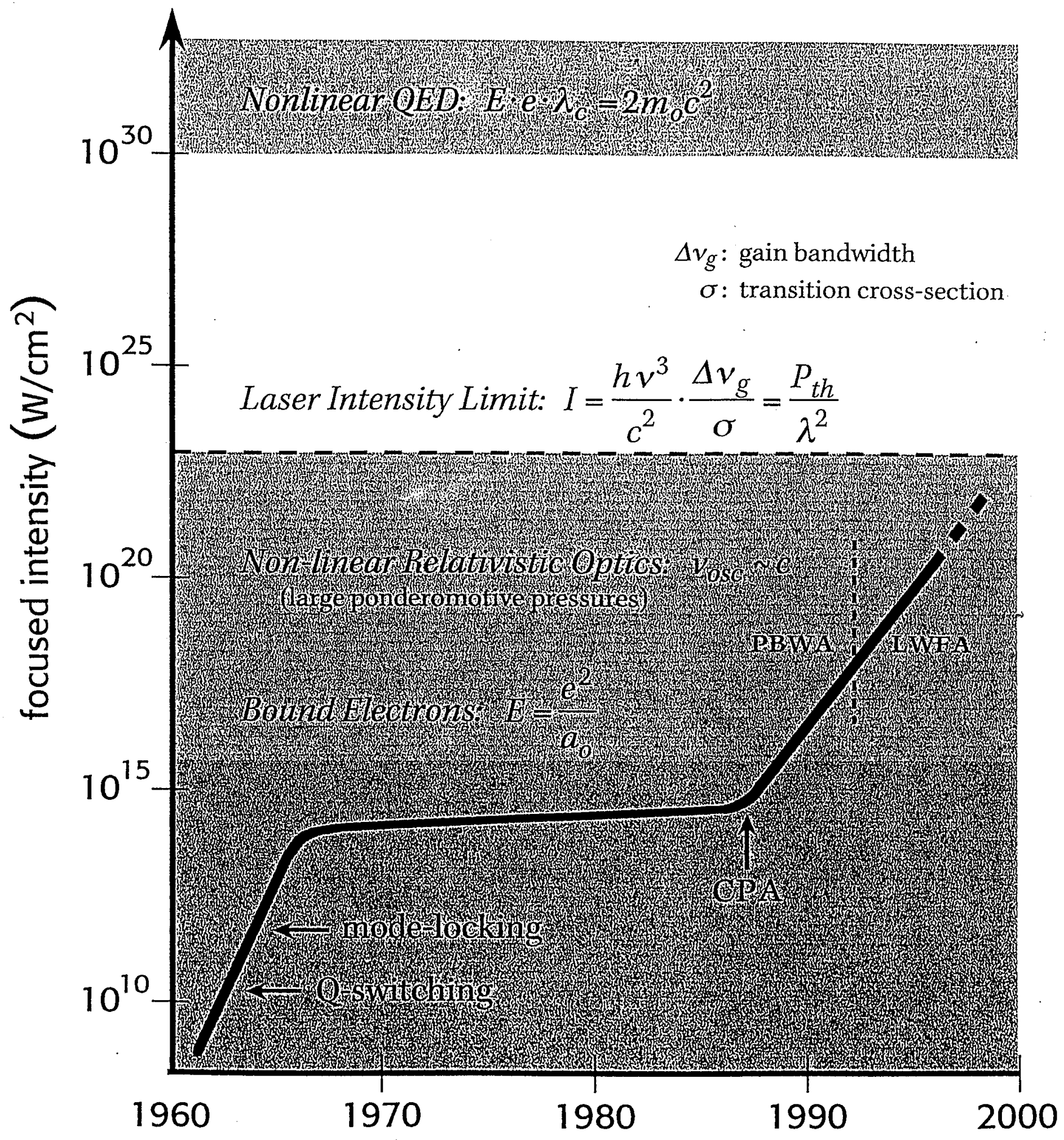




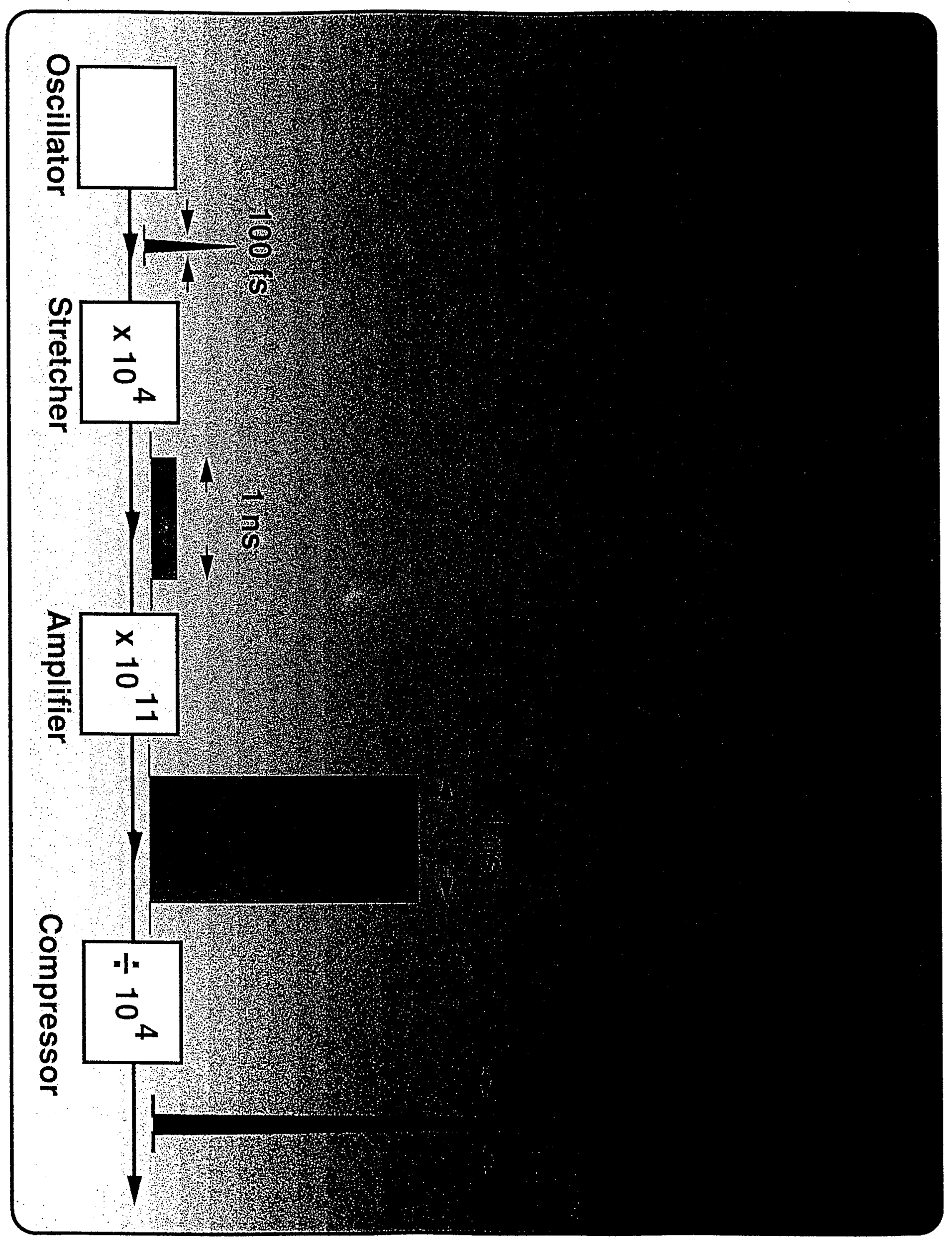



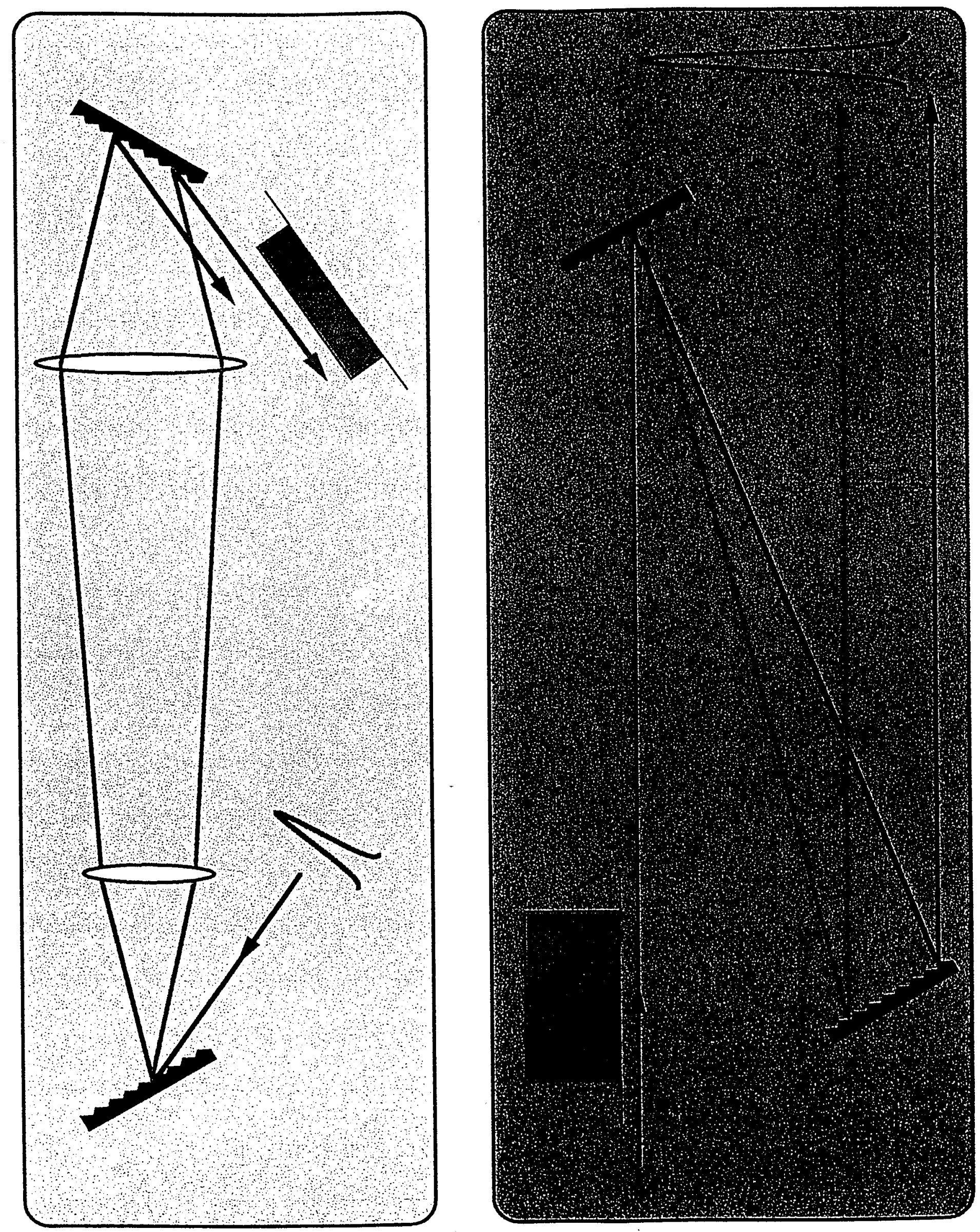


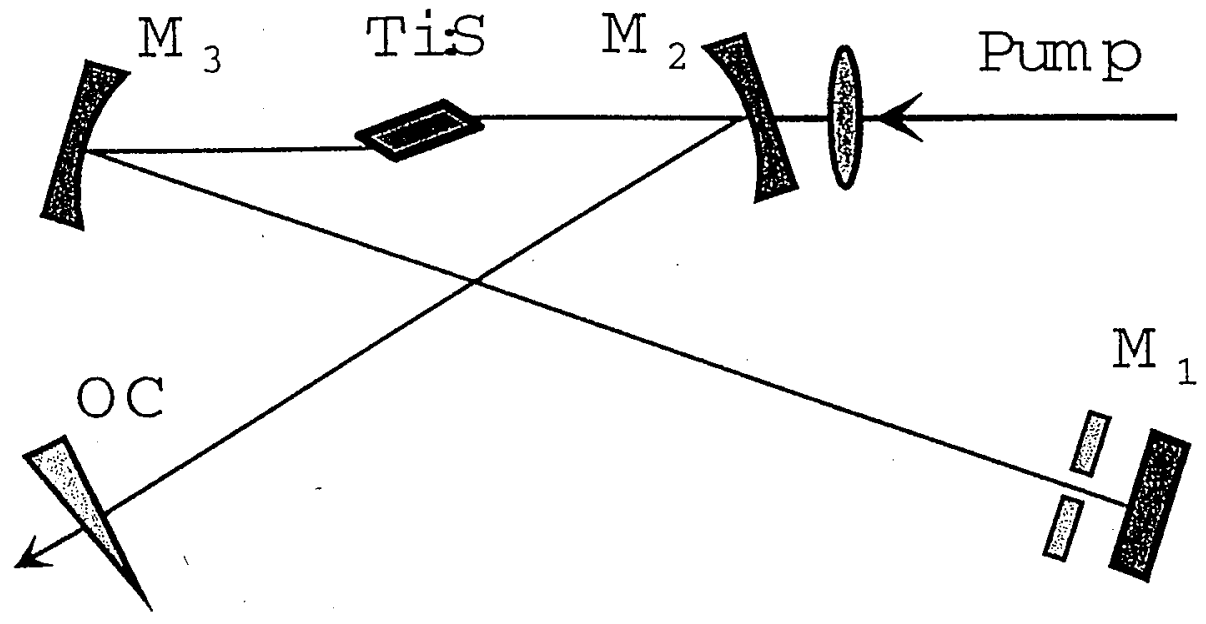




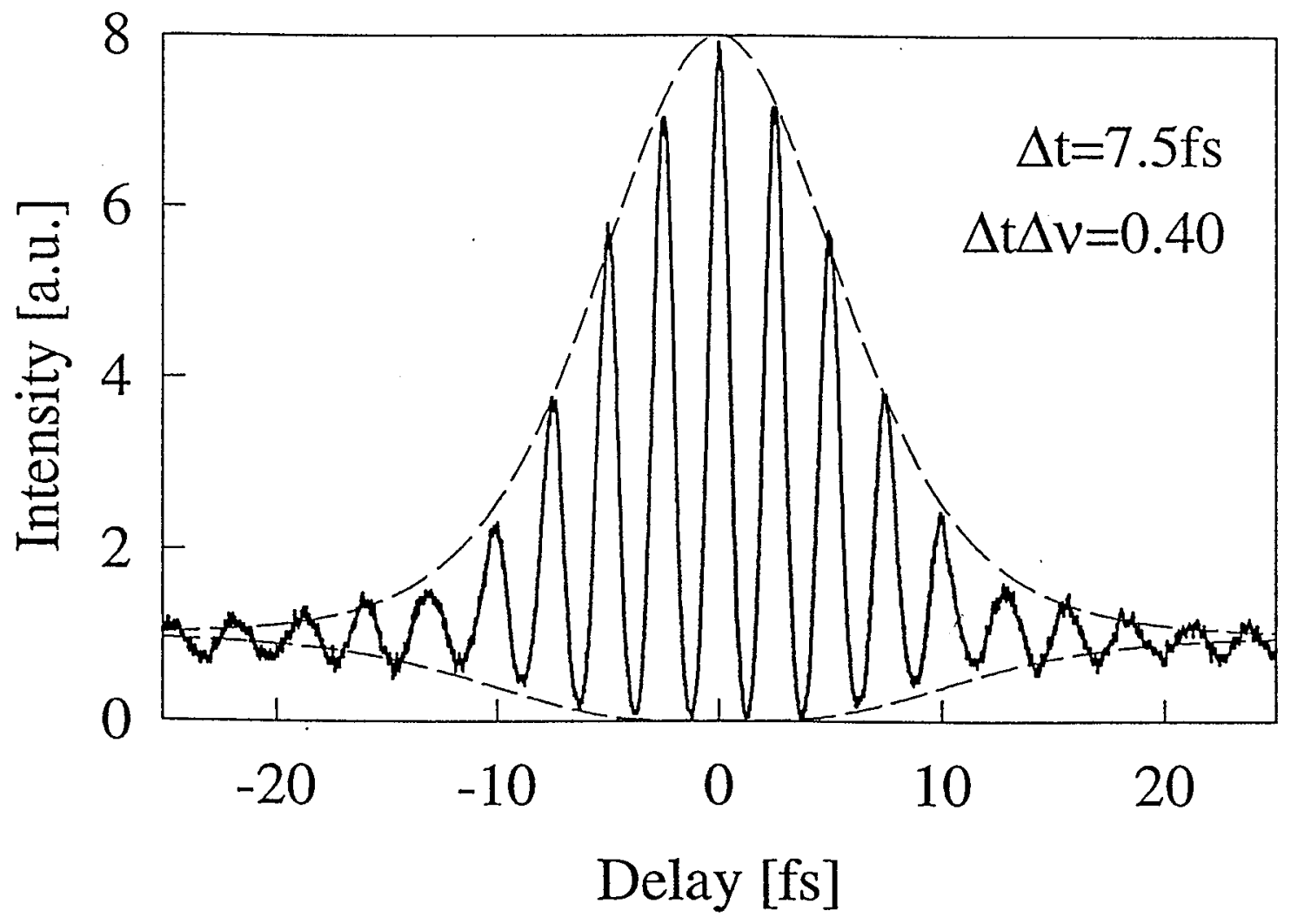




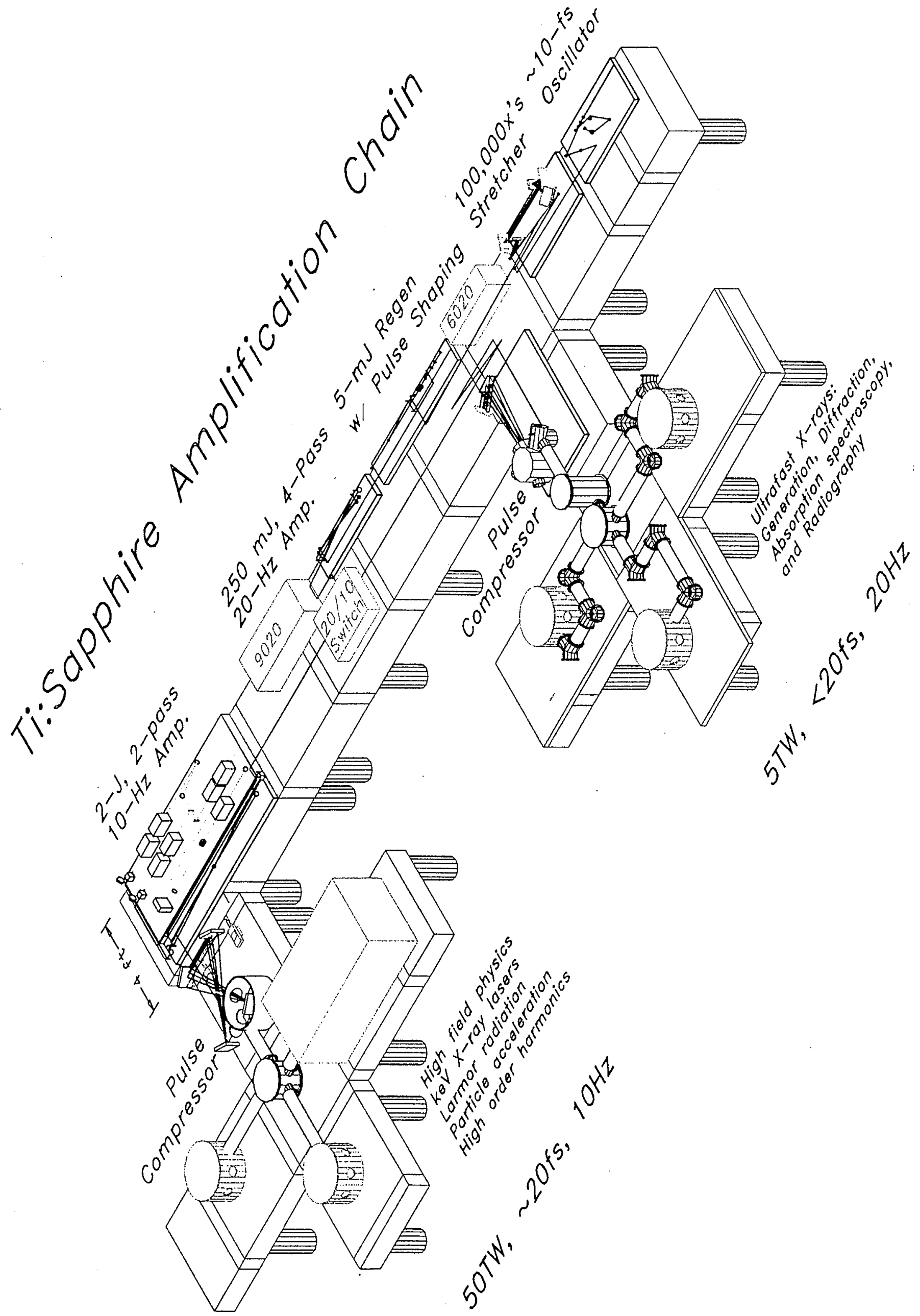




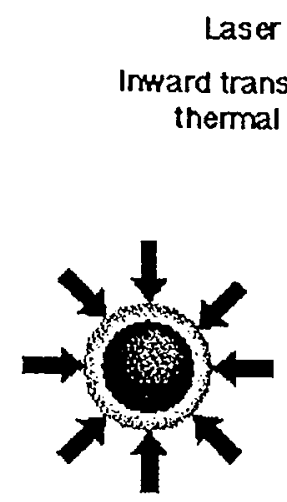

Atmosphere Formation

Laser or particle beams rapidly heat the surface of the fusion target forming a surrounding plasma emrelope
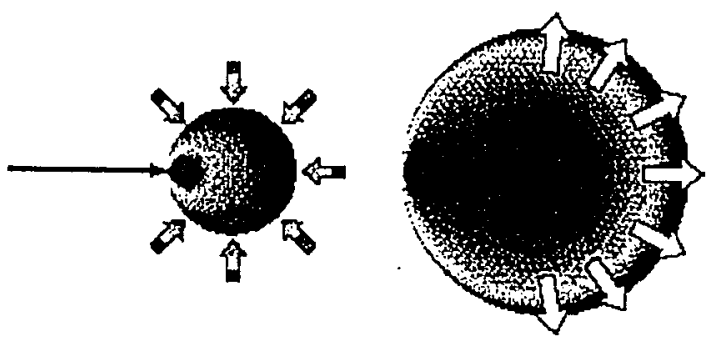

Ignition

At the moment of Ful is compressed by rocket-1ke blowoff of the surface material

$$
\begin{aligned}
& \text { maximum compression a } \\
& \text { short (1-10 psec) high } \\
& \text { intensity (1019 Whan }) \\
& \text { pulse ignites the capsule }
\end{aligned}
$$

Bum

Thermonuclear bum spreads rapidly through the compressedfuel, yleiding many times the driver input energy 


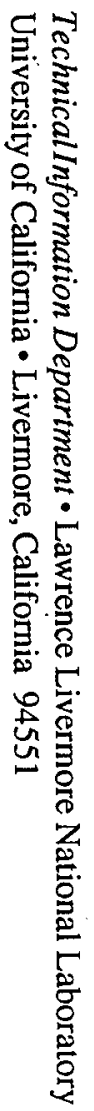

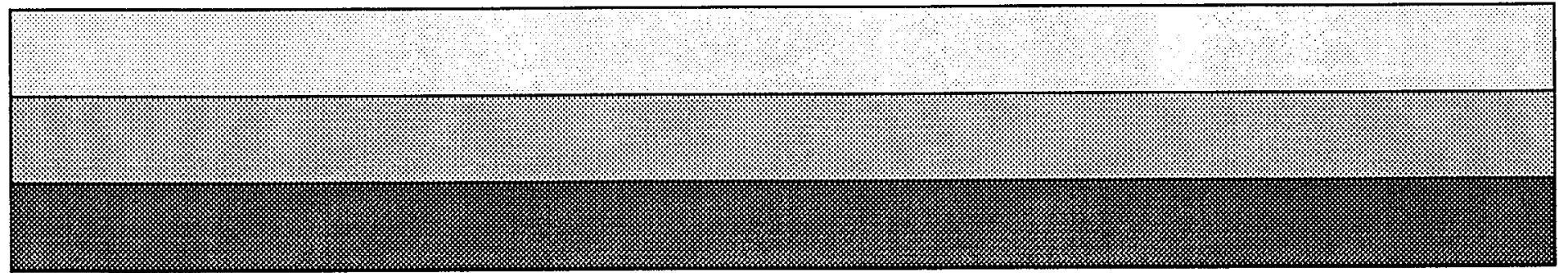

